\title{
ENJEUX ET DIMENSIONS
}

Véronique Mesguich, Julien Pierre, Camille Alloing, Gabriel Gallezot, Alexandre Serres, Richard Peirano, Fabrice Frossard, Christophe Deschamps, Michèle Battisti, Frédéric Martinet

A.D.B.S. | «Documentaliste-Sciences de l'Information »

2012/1 Vol. 49 | pages 24 à 45

ISSN 0012-4508

Article disponible en ligne à l'adresse :

http://www.cairn.info/revue-documentaliste-sciences-de-linformation-2012-1-page-24.htm

Pour citer cet article :

Véronique Mesguich et al., « Enjeux et dimensions », Documentaliste-Sciences de l'Information 2012/1 (Vol. 49), p. 24-45.

DOI 10.3917/docsi.491.0024

Distribution électronique Cairn.info pour A.D.B.S..

(C) A.D.B.S.. Tous droits réservés pour tous pays.

La reproduction ou représentation de cet article, notamment par photocopie, n'est autorisée que dans les limites des conditions générales d'utilisation du site ou, le cas échéant, des conditions générales de la licence souscrite par votre établissement. Toute autre reproduction ou représentation, en tout ou partie, sous quelque forme et de quelque manière que ce soit, est interdite sauf accord préalable et écrit de l'éditeur, en dehors des cas prévus par la législation en vigueur en France. Il est précisé que son stockage dans une base de données est également interdit. 
[ p.24 ] Le curateur, cet animal social dans la jungle informationnelle.

[ p.29 ] De la convergence des sphères publiques, professionnelles et privées

[ p.31] Curation et veille : des pratiques proches avec quelques différences fondamentales

[ p.33] Les deux faces de la curation scientifique

[ p.35] « Les outils de curation nous obligent à réinventer des mécanismes de confiance »

[ p.36 ] La curation à l'école, vers une culture de l'information

[ p.38 ] Curation, presse et journalistes, les liaisons dangereuses

[ p.40 ] Une offre foisonnante pour des usages émergents

[ p.44 ] La curation au risque du droit

[ p.45 ] « La curation d'aujourd'hui n'est pas celle dont je rêve ! »
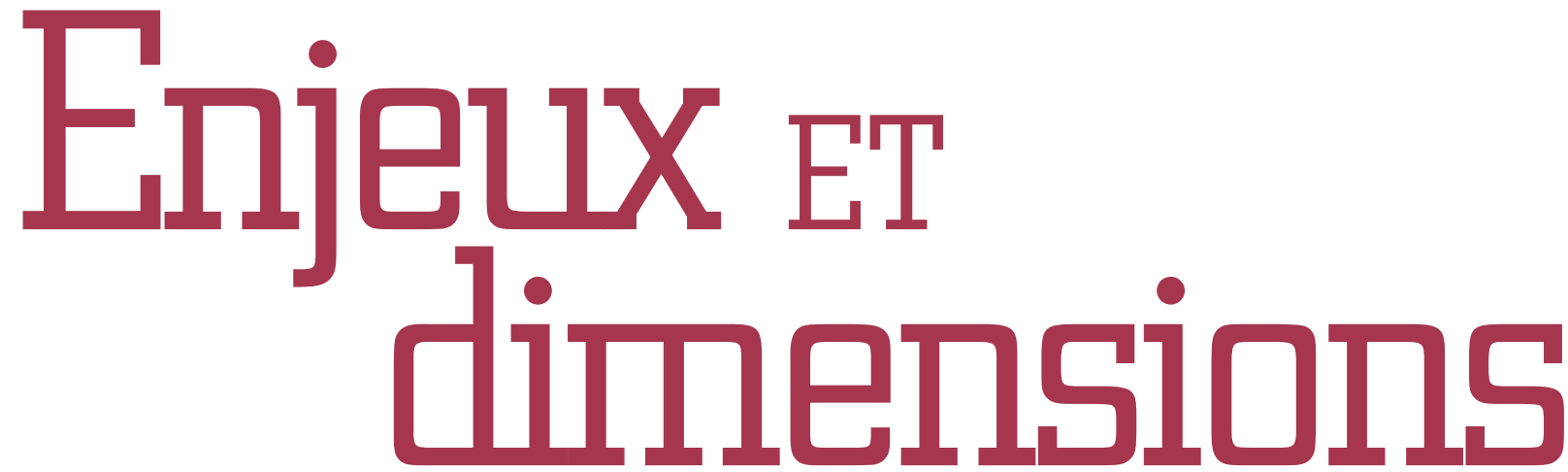

[ présentation ] Il en va de la curation comme de nombreux autres buzzwords ou néologismes à la mode : depuis son apparition à la fin des années 2000 , le terme fait l'objet de nombreuses discussions, tant dans le monde virtuel que dans le « monde réel ", pour être autant encensé que décrié. La curation serait-elle le dernier artefact du processus de redocumentarisation du monde numérique ? S'agit-il d'un nouveau métier, d'une pratique individuelle ou communautaire ? Cet article évalue les enjeux et dimensions de la curation, sur le Web ou au sein d'une entreprise, et ses liens avec la recherche classique d'informations.

\section{Le curateur, cet animal social dans la jungle informationnelle}

Le paysage informationnel qui, hier encore, offrait l'image d'un jardin à la française bien ordonné s'est transformé en une jungle dense, inextricable et luxuriante, sans cesse renouvelée. Cette surabondance génère moins un progrès technique du côté des outils de recherche qu'une évolution des usages de l'accès à l'information utile. Ce phénomène a été théorisé par les économistes: si la rareté d'une matière première engendre souvent une amélioration technique dans sa gestion, a contrario sa surabondance génère une mutation dans les usages. Nous sommes actuellement dans ce deuxième cas de figure. Les contenus numériques se multiplient, se diversifient et circulent de plus en plus vite via les plateformes de streaming, les applications pour mobiles et tablettes, les services d'échanges instantanés, etc. "The Web is dead » : sous ce titre un brin provocateur ${ }^{1}$, Chris Anderson formule 
l'hypothèse que ces nouvelles applications pourraient à terme venir concurrencer la navigation sur les sites web classiques... tout en prédisant une longue vie à l'Internet !

« L'homme est un animal social», affirmait le philosophe Aristote il y a plus de deux millénaires. Il est donc tout naturel que l'homo numericus soit devenu dans ce nouveau paysage un curieux animal social qui publie, partage, échange et enrichit des contenus numériques de tout type : " conversations », mais également liens vers des articles d'actualité, billets de blogs, références d'ouvrages, données sur le statut des personnes, fichiers multimédias, $\mathrm{CV}$, etc. Lidentité numérique se construit ainsi non seulement via une exposition d'informations et données personnelles, mais aussi à partir d'une pratique de sélection et partage de contenus en fonction de critères très subjectifs. Dans ce contexte, la curation consiste à repérer divers contenus numériques en fonction d'une thématique donnée, de sélectionner et filtrer les plus pertinents, de les organiser, les structurer à travers un dispositif de " scénographie » et d'en favoriser la diffusion.

\section{Naissance d'une nation de curateurs}

Une recherche sur le service Google Trends permet de dater à 2009 l'émergence de l'expression « content curation ». Mais dès 2008, le concept apparaît chez des blogueurs influents comme Steve Rubel, selon lequel les « digital curators » vont occuper une place importante dans l'avenir du Web, en élargissant aux contenus en ligne les fonctions traditionnelles des conservateurs de musées ${ }^{2}$. L'année suivante, le blogueur américain Robert Scoble ${ }^{3}$ fait appel aux start up prêtes à se lancer dans la curation en temps réel et y pressent une opportunité à « un milliard de dollars».

Lengouement pour la curation coïncide également avec la parution des premiers articles de Steve Rosenbaum, considéré comme l'un des pères fondateurs du concept. Cet entrepreneur original, PDG de la société Magnify, propose une plate-forme dédiée à la curation de vidéos en ligne. Il présente la curation comme « a key to information overload », une solution à l'infobésité et la surabondance informationnelle qui pourrait constituer l'avenir des médias et de l'édition: "The new media moguls won't be makers, they'll be finders, endorsers, and presenters ${ }^{4}$. Dans son ouvrage $e^{5}$, Steve Rosenbaum développe ainsi l'hypothèse que la curation deviendrait aussi importante que la création.

Le blog Masternewsmedia ${ }^{6}$, édité par « Robin Good » (pseudonyme de Luigi Canali de Rossi), consacre dès 2010 une série d'articles à la curation, présentée comme un remède face aux limites des moteurs web classiques. Le curateur y est décrit comme un curieux, passionné d'un sujet et désireux de

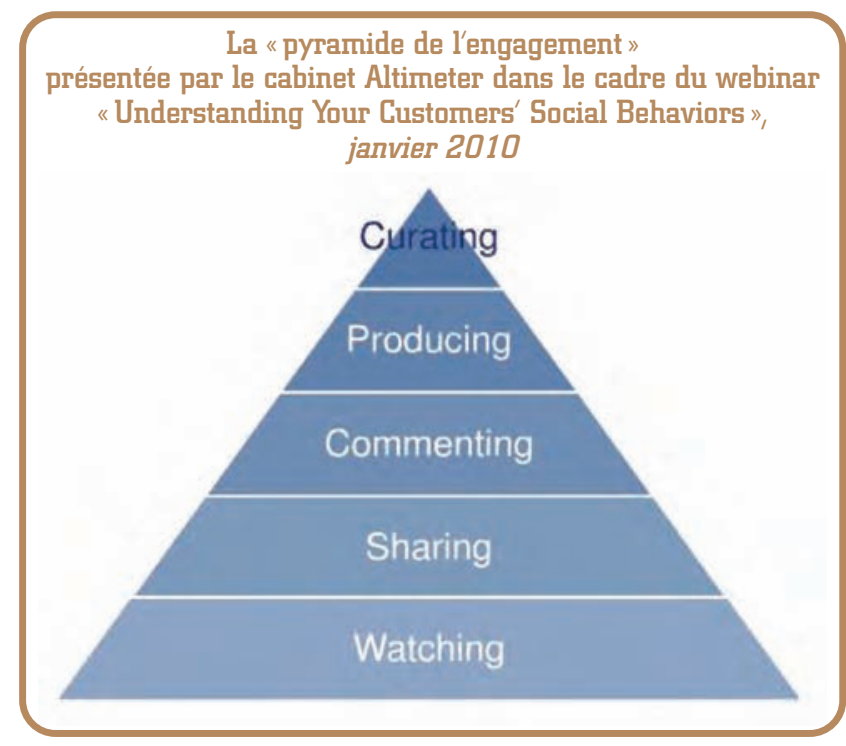

partager sa passion : une sorte de «DJ » de l'information numérique! Brian Solis, spécialiste des médias sociaux, va jusqu'à placer la curation au sommet d'une «pyramide de l'engagement » qui comprend plusieurs degrés : surveillance, partage, commentaire, production, curation (voir ci-dessus). mesguichveronique@yahoo.fir

Véronique Mesguich dirige I'Infothèque du pôle universitaire Léonard de Vinci. Elle enseigne également la maîtrise de l'information stratégique dans plusieurs établissements d'enseignement supérieur, dont l'École européenne d'intelligence économique, et anime régulièrement des sessions de formation continue autour de la méthodologie de recherche avancée et de veille sur Internet.

Lors de la conférence Web'10 qui s'est tenue à Paris fin 2010, la notion de curation été présentée comme une tendance émergente. Les services Scoop.it (créé par Marc Rougier et Guillaume Decujis) et Paper.li (développé par la start up suisse SmallRivers) étaient particulièrement à l'honneur, le second remportant même le prix de la viralité. Il n'en fallait pas plus pour lancer le buzz en France...
1 Chris Anderson, Michael Wolff. "The Web is Dead. Long live the Internet”. Wired, août 2010, http://www.wired.com/magazine/2010/08/ff_webrip

2 Cité par Elizabeth Schlatter, «A new spin. Are DJs, rappers and bloggers 'curators'? », Museum, January/February 2010, http://www.aam-us.org/pubs/mn/newspin.ffm 3 “The new billion-dollar opportunity: real-time-web curation", 22 septembre 2009, http://scobleizer.posterous.com/the-new-billion-dollar-opportunity-real-time 4 « Les nouveaux magnats des médias seront non plus les producteurs mais les personnes qui sauront trouver l'information, l'avaliser et la présenter». Steve Rosenbaum, “Are We a Curation Nation?”, Huffingtonpost, 8 mars 2010, http://www.huffingtonpost.com/steve-rosenbaum/are-we-a-curation-nation_b_ 489727.html

5 Steven Rosenbaum. Curation nation. Mc Graw Hill, 2011

$6 \mathrm{http}: / /$ www.masternewmedia.org/content-curation-and-the-future-of-search/ $7 \mathrm{http}: / /$ www.altimetergroup.com/2010/01/socialgraphics-webinar-slides-andrecording-now-available.html 


\section{/IIII/ Les 5 modèles de la curation}

Autre pionnier du concept de curation, Rohit Bhargava en donne une définition communément admise $^{8}$ : "L'action de trouver, regrouper, organiser et partager le contenu en ligne le meilleur et le plus pertinent sur un sujet spécifique. »Cependant, selon ce spécialiste du social marketing, le concept ne se définit pas uniquement comme une macro-activité englobant plusieurs pratiques différentes mais s'appuie sur cinq modèles : agrégation, distillation (dégager les éléments essentiels), élévation (extrapoler des tendances à partir de données partielles), mash-up (juxtaposer et fusionner des contenus) et organisation anté-chronologique.

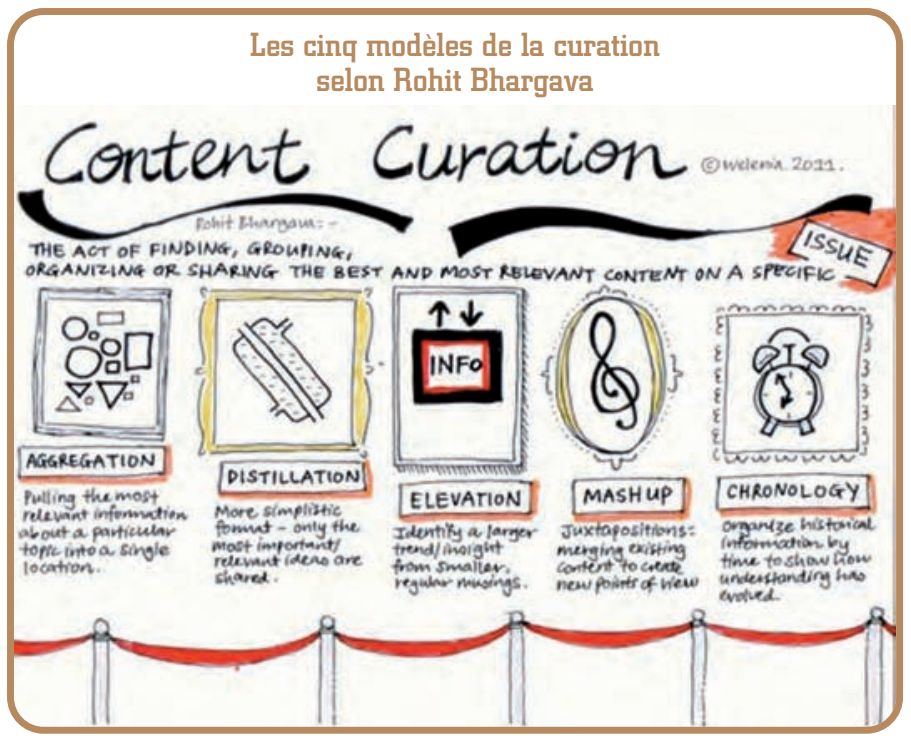

Ces cinq modèles sont à rapprocher des cinq types de filtrage définis par Tim Kastelle, spécialiste du management de l'innovation. Kastelle $e^{9}$ distingue le filtrage humain, fondé sur le jugement, et le filtrage mécanique, basé sur des heuristiques et des algorithmes. Le filtrage humain s'exerce à trois niveaux, allant du «naïf » à l'expert, en passant par le réseau. Notre intérêt et notre connaissance d'un sujet peuvent nous faire passer du statut de « filtreur naif » à celui d'expert. Comme l'énonçait Clay Shirky à la conférence Web 2.0 en 2008, le problème de l'accès à l'information n'est pas l'infobésité, mais «l'échec $d u$ filtre $»^{10}$. Lun des enjeux majeurs de la curation est donc bel et bien de mettre en place des filtres humains capables d'ordonner, de hiérarchiser et d'éditorialiser des contenus.

\section{Qui sont les « curateurs »?}

La notion de "gatekeepers » de l'information n'est pas nouvelle : traditionnellement, cette fonction est occupée en amont par les journalistes, éditeurs, enseignants, professionnels de la médiation informationnelle ou culturelle, etc. La facilité et l'immédiateté de l'accès aux sources d'information ont contribué à déplacer les rôles. Le filtrage s'effectue désormais davantage a posteriori, et par les internautes eux-mêmes, à travers leurs propres critères de choix. Selon le sociologue Dominique Cardon ${ }^{11}$, « le thème de la curation prolonge et radicalise la critique méfiante qu'adressent les citoyens à l'égard de l'autorité des professionnels de l'information».

Mais qui sont ces curateurs sur la Toile ? Sont-ils animés par un besoin de faire partager leurs passions dans un esprit altruiste, ou souhaitent-ils augmenter par ce biais leur présence sur le web ? Marc Rougier ${ }^{12}$, l'un des créateurs de la plateforme Scoop.it, estime que sa clientèle se compose pour moitié d'utilisateurs " personnels » qui créent des comptes consacrés à leurs sujets et hobbies favoris, l'autre moitié comprenant des utilisateurs « professionnels » dont les publications concernent des domaines d'expertise généralement transversaux. Il note que, pour ces derniers, " [...], l'usage est très varié, allant des très petites structures qui utilisent Scoop.it essentiellement pour contribuer à leur e-réputation, aux organisations qui utilisent Scoop.it pour de la veille/communication interne, aux grands groupes qui nourrissent leur présence web, y compris leur site corporate, de contenus issus de leur publication via Scoop.it. » On peut imaginer qu'au fil des années, la curation suivra une évolution analogue à celle du blogging : une banalisation et une érosion de la pratique, qui se concentrerait sur des usages d'experts.

Certains documentalistes, veilleurs ou responsables de CDI utilisent des plates-formes de curation pour agréger, organiser et diffuser des contenus à la manière d'une page publique Netvibes. Le CDI du collège savoyard George Sand a ainsi créé plusieurs comptes Scoop.it consacrés à l'innovation pédagogique ${ }^{13}$ ou à l'actualité des professeurs-documentalistes ${ }^{14}$.

En entreprise, le curateur pourra exercer au sein d'un réseau social d'entreprise, alimenter des communautés avec des contenus ciblés selon les thématiques, faire émerger les signaux faibles et accompagner le changement.

Pour les professionnels de l'information, la curation peut ainsi être considérée comme le « quatrième $C$ » à ajouter aux autres facteurs de création de valeur:

- création de contenus (analyse ou synthèse de documents)

- communication (diffusion d'information ciblée en fonction des besoins)

- communauté (animation de communautés ciblées et dissémination sélective de contenus).

Les professionnels du marketing, eux, voient en la curation une opportunité pour promouvoir une marque et améliorer la gestion de l'e-reputation et du brand content.

La curation est également un atout pour les référenceurs professionnels : en effet, les plateformes comme Storify ou Scoop.it font l'objet d'un bon référencement naturel par les robots des moteurs. À partir d'un compte, une personne physique ou morale, ou encore une marque, peut ainsi être facilement identifiable comme expert dans un domaine et gagner en visibilité.

Le curateur est parfois présenté comme un « pilleur $»^{15}$ qui aurait tendance à s'approprier, éditorialiser et diffuser en son nom des contenus créés par des tiers. Jacques Breillat préfère le considérer comme un « aiguilleur du web $»^{16}$, dont l'expertise consiste à sélectionner l'information pertinente et la diffuser en juste à temps aux personnes concernées. 
On retrouve dans ces différents profils de curateurs la figure du « Maven », telle que définie dans l'ouvrage de Malcolm Gladwell ${ }^{17}$. Le Maven est un collectionneur et diffuseur intensif d'informations et d'impressions, ce qui lui permet d'être le premier à détecter ruptures et tendances et de pouvoir exercer une influence sur les membres de sa communauté.

\section{La convergence des continents}

Si la physionomie du web se modifie, le principe de fonctionnement des moteurs de recherche, leurs options d'interrogation et de restitution des résultats ont assez peu changé en revanche au cours de ces dernières années. Les grands moteurs continuent de diversifier leur offre dans un marché saturé et de fidéliser leurs utilisateurs en proposant de nouveaux services : recherche « sociale », temps réel, recherche multimédia, etc. Dans ce contexte, la curation de contenus constitue une approche alternative, basée moins sur la dimension technique que sur les interactions sociales.

Rappelons que le moteur Google a été conçu en 1998, à une époque où le Web était encore relativement linéaire et majoritairement textuel. Ses plus proches concurrents, à savoir les moteurs Yahoo!Search ou Bing sont apparus ultérieurement mais restent bâtis sur le même modèle : robot de collecte, module d'indexation, interface d'interrogation, et algorithmes de pertinence. L'un des facteurs clés du succès de Google a été, entre autres, la création du fameux algorithme Page Rank basé sur l'analyse automatisée du nombre de liens, et la provenance de ces liens, l'objectif étant essentiellement de donner une meilleure visibilité aux sites de « référence » dans un secteur donné. Cette idée révolutionnaire était particulièrement adaptée à la structure du Web à l'époque : elle l'est moins aujourd'hui. Bien entendu, les moteurs actualisent régulièrement leurs algorithmes en fonction de l'évolution de la forme et des contenus du Web. Google a ainsi introduit en France à l'été 2011 une mise à jour importante de son algorithme, sous le nom de "Panda ». Il s'agit en effet de cibler la qualité des résultats et d'éliminer des sites ayant un contenu trop pauvre ou redondant : sont notamment visées les «fermes de contenus» résultant de l'agrégation de liens et contenus extérieurs. Dans le même temps, les algorithmes de référencement des moteurs commencent à prendre en compte les « votes » des internautes à partir des boutons Like de Facebook ou +1 de Google. On voit ainsi que les réseaux sociaux vont avoir de plus en plus d'impact dans le classement des résultats, et donc dans le référencement naturel.

Il est intéressant de constater un double phénomène d'évolution et de convergence des géants rivaux Google et Facebook. Le moteur, qui a du propre aveu de ses dirigeants raté son entrée dans le monde des médias sociaux, tente de rattraper son retard avec son nouveau réseau Google Plus, lancé à l'été 2011. Un peu plus tôt, au printemps de la même année, Facebook obtenait un brevet (Visual Tags For Search Results Generated from Social Network Information) fondé sur le principe de la curation. Le site Abondance en révélait quelques éléments en mars dernier : « Les résultats organiques d'un moteur de recherche seraient alors basés en partie sur la fréquence de clics des usagers du réseau social $»^{19}$. Le système pourrait être appliqué aux résultats du moteur Bing dans l'avenir.

Les plateformes « 2.0 » dépassent ainsi la simple fonction d'échange et acquièrent des fonctions de recherche et de découverte de contenus informationnels. Ces découvertes ne sont pas le fruit d'un hasard fortuit, mais sont favorisées par la taille et la diversité du réseau social : les découvertes s'effectuent en fonction de ce que les membres partagent en ligne, une forme de « sérendipité sociale » selon Henry Nothaft ${ }^{20}$. Nous passerions ainsi d'un archétype de la recherche classique à un modèle basé davantage sur le hasard des découvertes inattendues, via des contenus partagés ou recommandés par les internautes. Après plusieurs années de domination du modèle classique à la Google, les « outils froids » fondés sur des algorithmes se rapprocheraient des « outils chauds » basés sur le relationnel et la médiation humaine.

\section{Veille, sourcing et curation}

Nous l'avons vu, l'expression « curation de contenus » a fait tout récemment irruption dans le champ sémantique du Web. Pour autant, les fonctions de sélection, partage et organisation existent déjà dans bon nombre de plateformes du Web 2.0. Les solutions de curation viennent ainsi compléter une panoplie d'outils de sourcing et d'agrégations de contenus :

- le bookmarking social permet d'identifier des sources pertinentes et de qualité en se reposant sur l'expertise des contributeurs de plateformes comme Diigo ou Pearltrees. Ce dernier, caractérisé par une interface graphique très riche, se définit

8 "Manifesto For The Content Curator: The Next Big Social Media Job of The Future ?", 30 septembre 2009, http://rohitbhargava.typepad.com/weblog/2009/09/manifestofor-the-content-curator-the-next-big-social-media-job-of-the-future-.html 9 Tim Kastelle, "Five forms of filtering”, avril 2010,

http://timkastelle.org/blog/2010/04/five-forms-of-filtering

10 "It's Not Information Overload. It's Filter Failure", Web 2.0 Expo, New York, 18 septembre 2008, http://www.youtube.com/watch?v=LabqeJE0Qyl\&feature=youtu.be 11 « Tous éditeurs ? Les promesses incertaines de la « curation », 26 avril 2011, http://cblog.culture.fr/2011/04/26

12 L'Interview BlogoStart'up : Marc Rougier pour Scoop.it, 12 novembre 2011, http://www.blogoergosum.com/29261-linterview-blogostartup-marc-rougier-pourscoop-it-2

$13 \mathrm{http} / / /$ www.scoop.it/t/pedagogie-et-nouveautes-disciplinaires

$14 \mathrm{http}: / /$ www.scoop.it/t/l-actu-des-cdi-et-des-profs-docs

15 Voir par exemple le blog d'Anthony Poncier http://poncier.org/blog/?p=2697

16 Voir : « Agrégateurs de contenus : halte au plagiat ! », Amaranthe, 11 sept. 2011, http://www.amaranthe.be/blog/publication/agregateurs-de-contenus-halte-auplagiat

17 « Curateur digital : les aiguilleurs du Web ! », 27 mai 2011,

http://jacques.breillat.fr/concepts/curateur-digital-les-aiguilleurs-du-web

18 Malcolm Gladwell, Le point de bascule: comment faire une grande différence avec de très petites choses. Montréal : Éditions Transcontinental, 2003 (Collection Commerce) 19 Olivier Andrieu, « Facebook obtient un brevet associant la curation à la recherche d'information », Abondance, 18 mars 2011,

http://actu.abondance.com/2011/03/facebook-obtient-un-brevet-associant-la.html 20 Henry Nothhaft, "The Myth Of Serendipity", TechCrunch, 27 nov. 2010, http://techcrunch.com/2010/11/27/myth-serendipity/ 
/////// d'ailleurs à la fois comme un site de bookmarking social et de curation ;

- les pages publiques Netvibes rassemblent des flux RSS provenant de plusieurs sources ou des widgets spécialisés ;

- peu connu en France, le « moteur de découverte » StumbleUpon permet d'effectuer des recherches par « similarité », la ressemblance étant calculée statistiquement à partir des usages ou bookmarks des usagers :

- l'agrégation d'actualités : le journaliste Tom Foremski ${ }^{21}$ voit une différence majeure entre curation et agrégation. Selon lui, la curation est indissociable de la notion de critères de sélection, contrairement à l'agrégation qui se limite à une juxtaposition de contenus. Le site américain Digg, lancé dès 2004, est devenu emblématique des " digg like » (Reddit, Scoopeo, par exemple), sites d'agrégations d'actualités dont le système de hiérarchisation des contenus (articles, blogs, vidéos, etc.) est assuré par le vote des utilisateurs. Le « digg like » francophone Wikio News a malheureusement cessé son activité fin 2011 ; - le principe du journalisme de liens, ou « journalisme augmenté », est également proche de la curation : des blogueurs ou journalistes sélectionnent des articles ou billets pour leur pertinence ou leur intérêt et rassemblent les liens vers ces contenus sur des plateformes comme Aaaliens, Owni ou Techmeme.

Pour Brian Solis, cité plus haut, le principe du hashtag sur Twitter constitue une forme de curation, dans la mesure où cette pratique facilite les tris et optimise l'accès aux contenus pertinents. La nouvelle présentation des profils Facebook sous forme de « journal » (Timeline) qui se généralise actuellement illustre également les principes de Rohit Bhargava évoqués plus haut. On voit ainsi que le principe de la curation n'a rien de révolutionnaire mais vient faire converger et se superposer de nombreuses pratiques déjà existantes.

\section{Ouverture ou enfermement?}

Les exemples de sites sociaux mentionnés plus haut, de même que les plateformes de curation, constituent pour le veilleur des moyens d'identification et de découverte de sources ou contenus originaux. La valeur ajoutée de tous ces outils réside dans la qualité de la sélection effectuée par des humains, dans le partage des sources ainsi que dans l'effet de levier de la communauté. De même, les risques y sont similaires : redondance de l'information d'où le risque d'augmentation fictive de certains signaux forts au détriment de véritables signaux faibles; homophilie et enfermement dus à une trop grande proximité relationnelle.

Plusieurs études récentes donnent des résultats parfois contradictoires à ce sujet et ont tendance soit à confirmer, soit à infirmer ce risque de
« rétrécissement » de l'espace informationnel personnel, qui irait à l'encontre de la sérendipité. Une étude menée en $2010^{22}$ prouve l'importance dans un réseau social des « liens faibles » (par exemple, amis avec qui l'interaction est faible) qui véhiculeraient en effet l'information réellement nouvelle et originale pour un internaute. Pour autant, cette notion d'information « nouvelle » pour un utilisateur est extrêmement subjective et la notion de signal faible n'a pas d'existence intrinsèque.

Comme le montre Camille Alloing (voir article ci-après) la curation est proche des pratiques de veille, mais peut-on l'appliquer efficacement à tous les types de veille? La veille technologique, qui met en œuvre de nombreuses sources du Web « visible » (articles, blogs d'experts, portails spécialisés, etc.), se prête tout particulièrement à la pratique de la curation. De nombreux comptes Scoop.it sont d'ailleurs consacrés à des applications de veille sur des technologies émergentes : open source, énergies vertes, réalité augmentée, etc. La surveillance de l'e-reputation, via les relais d'opinions et blogs d'influenceurs, peut s'appuyer également sur la curation. En revanche, celle-ci semble moins adaptée à la veille concurrentielle ou juridique, où l'on recourt davantage aux sources du Web « invisible », comme les bases de données accessibles sur abonnement payant.

\section{L’ingénieur et le bricoleur}

Lanthropologue Claude Levi Strauss ${ }^{22}$ distingue d'une part l'ingénieur, représentant du monde moderne qui impose sa vision, sa maîtrise des technologies et son projet, et d'autre part le bricoleur, adapté à son écosystème grâce à sa connaissance du terrain et son aptitude à se débrouiller avec « les moyens du bord".

Le professionnel de l'information procèderait ainsi en ingénieur, fort de sa science des outils documentaires classiques, des requêtes complexes et de la maîtrise des sources d'informations structurées. Le curateur, lui, pourrait incarner la figure du bricoleur de part son agilité à repérer les contenus utiles, les agréger et les diffuser via des plates-formes souples et évolutives, nées des vagues successives de la courte histoire d'Internet. À l'époque des "grandes découvertes » du Web, les pionniers des outils de recherche s'appelaient Copernic ou Magellan. Les annuaires de recherche, aujourd'hui malheureusement quasiment disparus, peuvent être considérés a posteriori comme des outils de curation avant la lettre. Les modes évoluent vite sur le Web : même si le buzzword « curation » risque d'être rapidement démodé, la pratique, elle, n'est pas nouvelle et répond à de véritables besoins, avec un positionnement original : au confluent de la recherche classique, du tagging collaboratif, de la veille et du knowledge management. •

21 Tom Foremski, “Curation versus aggregation represents human web versus machine web...”, Zdnet, 3 novembre 2010,

http://www.zdnet.com/blog/foremski/curation-versus-aggregation-representshuman-web-versus-machine-web/1569

22 Eytan Bakshy, Itamar Rosenn, Cameron Marlow, Lada Adamic, The Role of Social Networks in Information Diffusion, janvier 2012, http://arxiv.org/abs/1201.4145 23 Claude Levi Strauss, La Pensée sauvage, Paris: Presses Pocket, 1990 


\author{
[ tendance ] Comment, en se posant la question du \\ coup de cœur du professionnel de l'information, le \\ phénomène de la curation met en lumière un certain \\ nombre d'évolutions sociologiques.
}

\section{De la convergence des sphères publiques, professionnelles et privées}

Pour commencer, une question entendue en formation dans une URFIST : "Et pourquoi le documentaliste ne pourrait-il pas, comme le libraire, rendre public ses coups de coeur ? » Il est vrai qu'il existe déjà une économie de cette publicité, via les têtes de gondole dans les espaces culturels marchands ou les sélections à l'entrée des bibliothèques. Cette question va nous servir de toile de fond pour placer la curation à l'intersection de différents espaces sociaux.

Que l'on se fie à un agent-logiciel ou à un professionnel de l'information, la curation - entendue autant comme mécanisme informatique que comme pratique sociale - fait reposer l'économie de la recommandation sur l'individu, de l'imaginaire sociotechnique qui va nourrir le concepteur des algorithmes aux choix que fera le gestionnaire du service web en décidant ou non de mettre en avant telle ressource. Dès lors, on peut se demander en quoi la part de subjectivité interfère avec une décision d'ordre professionnel : quelle place accordée dans une démarche documentaire à ce qui relève des sentiments et de la vie affective? Dans quelle mesure cette dernière nourrit-elle notre travail ? Plus largement, cela pose la question de la frontière entre vie privée, espace professionnel et sphère publique.

\section{Découper les espaces}

Le modèle sociopolitique de la tradition philosophique grecque distingue clairement l'oïkos et la polis. Le maître de maison, à l'intérieur de laquelle il dispose d'une liberté de corps, prouve par son titre de propriété sa capacité à se débarrasser des contingences matérielles : il est dès lors habilité à participer à la gestion de la cité, au sein de laquelle il dispose d'une liberté de parole. Ce qui se déroule à l'intérieur de l'oïkos peut donc y rester cantonné ; pire encore, la maison est le lieu de privation du plaisir d'être dans l'espace public : être dehors, c'est ex-ister (Arendt). De même, parmi les activités domestiques se trouvent des formes de production matérielles, ainsi tout ce qui relève du professionnel est aussi à ranger dans la catégorie de l'espace privatif : au jardin secret de la vie privée s'ajoute le secret professionnel. Cette séparation entre oïkos et polis, entre privé et public va tenir bien après l'émergence de l'espace public politique bourgeois identifié par Habermas: le bourgeois dispose des mêmes privilèges que le maître de l'oükos, à savoir disposer d'une vie privée pour disposer d'un agir communicationnel dans l'espace public, sans pour autant que soit publié ce qui se déroule en privé.

À cette première catégorisation philosophique, nous pouvons ajouter celle du dualisme entre le corps et l'esprit (Descartes), le premier relevant de l'intime et le second se produisant sur un mode rationalisant, qui exclut donc la dimension affective du sujet pensant. Le processus délibératif qui se déroule au sein de l'espace public prend ainsi une forme discursive basée sur une argumentation rationnelle. La seule dimension personnelle qui a droit de cité est attachée à l'identité et à ses titres : état-civil, classe sociale, diplômes, place dans l'organisation, etc.

Un courant sociologique montre comment les pulsions ont été affinées dans la sociogenèse occidentale (Elias), comment les gestes ont été calculés dans les relations interindividuelles (Goffman), et comment le capitalisme a transformé les émotions en marchandise ${ }^{1}$.

\section{Décloisonner}

Le modèle sociopolitique moderne propose justement de construire une société d'indistinction (république égalitaire où ceux qui étaient relégués dans l'oïkos ont dorénavant le droit de s'exprimer - et d'exister - en public et où les signes discriminants 
/////// sont interdits par la loi) tout en caractérisant l'individu par son identité et par l'injonction moderne à être « soi » (seule possibilité d'être dans une société de masse $)^{2}$. Un double effet de transparence est alors produit :

- la démocratie repose sur la publicité (et l'archivage) des trois pouvoirs juridique, législatif et exécutif (dynamique de l'open data versus impossibilité d'un droit à l'oubli), même si parfois cette publicité est forcée (productions journalistiques ou associations de type regardscitoyens.fr ou wikileaks.org). Les activités économiques sont également soumises à une forme de transparence : publicité des comptes, des contrats de travail et, dans un sens plus commercial, publicité sur les produits. Plusieurs dérives naissent de la convergence de ces domaines politiques, marketing et informationnels : affichage de la vie personnelle des personnages politiques, violation de secrets d'État, atteinte à la réputation ; - à un niveau individuel, les dérives semblent les mêmes. Ce besoin de parler de soi que le psychiatre Serge Tisseron nomme « extimité » peut conduire à des errements : intense exposition médiatique, même dans le cercle restreint d'une cour d'école ou de bureaux ; sentiment, dès lors qu'il n'y a plus de privilèges, qu'il n'y a plus de vie privée ${ }^{3}$, que le décloisonnement est achevé et que se sont effacées les frontières entre vie privée et vie publique ${ }^{4}$. Alors qu'il ne s'agit dans un sens - psychiatrique - que d'une phase nécessaire au développement de (l'estime de) soi et dans l'autre - sociologique - d'un moyen d'être identifié par son groupe de référence.

\section{Être affecté}

En outre, nous portons aujourd'hui un nouveau regard sur notre rapport au corps. Le dualisme se fait aussi sentir au niveau épistémologique : la place du corps dans les processus intellectuels n'a que peu été étudiée. Pourtant, on doit à des courants comme le constructivisme (Piaget) ou la pragmatique (Bateson) l'identification d'une communication non verbale, ou encore d'une articulation entre l'affectif et le cognitif : l'idée d'une incorporation des processus de symbolisation et la part déterminante que peut tenir le corps face à l'esprit.

Évidemment, il faut aussi prendre en compte les progrès réalisés par l'imagerie médicale, qui ont fait ressortir l'activation de zones cérébrales selon l'activité mentale de l'individu. On sait notamment depuis les travaux du neurologue A. Damasio le rôle que les émotions jouent dans la construction du raisonnement. Ainsi se sédimentent des travaux ayant d'abord une portée thérapeutique avant d'en arriver à une dimension psychosociologique.

\section{Du " care " à la curation}

Dès lors que convergent des processus affectifs et décisionnels, il faut s'attendre à voir converger également des phénomènes issus de sphères sociales distinctes: la vie affective, l'espace professionnel, le marché et la sphère publique s'irriguent mutuellement. Rien de bien nouveau, comme le signale I. Berrebi-Hoffman ${ }^{5}$ : «Force est de constater que, dans nos mondes contemporains, il y a de l'intime dans le travail, de l'intervention de l'État et de la sphère publique dans les familles, et de l'intime convoqué dans la sphère publique et politique. Les frontières entre le public et l'intime se redéfinissent »

Trois questions pour finir ce rapide parcours historique et notionnel : si l'on reconnaît la perméabilité des sphères privative, professionnelle et publique, cela suffit-il pour autant à autoriser le coup de cœur documentaire en entreprise ou sur le Web ? Et cette approche correspond-elle à ce qu'on range dans la pratique de la curation? Enfin, dans quelles mesures ces considérations déplacent-elles le problème sur l'identité du curateur?

Les nouvelles pratiques sociales liées à l'information numérique et aux services web ont largement été étudiées dans le champ scientifique et il a été montré en quoi le statut d'auteur était redéfini, interrogeant la légitimité d'un amateur s'exprimant dans un espace professionnel ou public (voir par exemple les travaux d'Évelyne Broudoux ou le texte d'Olivier Le Deuff dans ce dossier).

Le point de convergence des nombreuses définitions de la curation est l'idée d'une sélection parmi la masse informationnelle collectée par le veilleur. Soit l'on considère la curation comme une réorganisation d'un catalogue (ce que font les museum curators) autour d'un axe de pertinence, et l'on s'interrogera alors sur les critères de sélection employés (y compris à travers un algorithme), considérant que cette approche rationnelle est motivée (c'est-à-dire mue par des émotions). Soit l'on considère la curation comme une éditorialisation autour d'un centre d'intérêt, auquel cas ce sont les éléments de la vie affective qui sont soumis à rationalisation.

Roger T. Pédauque explique en quoi « le privé est publicisé » dans la mesure où les documents sont des « objets transitionnels » entre des espaces de communication privé et public, tout à la fois espaces de production et de réception ${ }^{7}$. Dans ce sens, nous pensons que, après les blogs ou la gestion de la réputation dont parlait déjà Pédauque et dans lesquels il voyait une hybridation des pratiques documentaires, la curation pourrait être une pratique professionnelle de redocumentarisation enrichie non pas tant par des métadonnées que par des éléments relevant de la sphère privative et de la vie affective.

2 Voir les intellectuels contemporains traitant de l'individu : P. Ricœur, C. Taylor, U. Beck, A. Giddens, C. Lasch, Z. Bauman, A. Ehrenberg, J.-C. Kaufman, M. Castells, etc. 3 D. Boyd, "Privacy is a Privilege", Apophenia, 21 juillet 2005,

http://www.zephoria.org/thoughts/archives/2005/07/21/privacy_is_a_privilege.html 4 Comme le revendiquent les dirigeants de Google (E. Schmidt) ou de Facebook (M. Zuckerberg).

5 I. Berrebi-Hoffmann, « Les métamorphoses de l'intime », Empan, 2010, vol. 1, n 77, p.13-17, http://www.cairn.info/revue-empan-2010-1-page-13.htm

6 R. T. Pédauque, « Document et modernités », in : R. T. Pédauque, Le Document à la lumière du numérique. Forme, texte, médium : comprendre le rôle du document numérique dans l'émergence d'une nouvelle modernité, C\&F Éditions, 2006 7 R. Odin, Les espaces de communication : introduction à la sémiopragmatique, Grenoble : PUG, 2011 


\section{Curation et veille : quelques différences fondamentales}

Si la curation a été l'un des mots-clés de 2010, c'est que, comme souvent sur le Web, ce concept ne fait qu'agréger des pratiques déjà existantes par ailleurs. Sous un habillage marketing ayant permis la création de nombreuses plates-formes dédiées, la curation n'est pas sans rappeler les pratiques de veille et de gestion du document classiquement utilisées par les professionnels de l'info-doc. Seulement, et c'est là l'objet de cet article, un «curator » n'est pas un veilleur, et inversement. La limite entre les deux pratiques est ténue, et il paraît intéressant d'en tracer les frontières pour mieux en préciser les rôles.

Tout d'abord, et très succinctement, quid du curator et de la curation ?

Pour les Anglo-saxons, un curator est le responsable des collections d'un musée. Par définition, il " prend soin » des œuvres dont il a la charge, il les conserve, les archive, les sélectionne pour une exposition, les met en valeur, etc. Transposé au monde du Web et de l'information, le curator devient donc celui qui identifie les sources, trie et collecte les informations, agence les contenus puis les diffuse (il les « expose ») sur les réseaux ou communautés auxquels il appartient. En terme d'usages, il fait partie des 20\% d'internautes désignés comme « collectors » par le cabinet Forester ${ }^{1}$; par extension, chaque utilisateur d'un réseau social (on peut notamment penser à Twitter), d'un blog ou d'une plate-forme UGC ${ }^{2}$ en général est un curator en devenir, à partir du moment où il choisit de diffuser des contenus thématiques.

Jusqu'ici donc, il opère de la même manière qu'un veilleur : choix des sources, de l'information et de son mode de diffusion. Tout comme les curators, le veilleur dispose souvent d'un accès à une plate-forme dédiée (ou une base de données) pour diffuser les résultats de sa veille. Tout comme le curator, le veilleur utilise des outils lui permettant la collecte d'informations, outils dont il est dépendant quant à ses capacités de collecte, de traitement et de diffusion.

Si nous parlons maintenant d'objectifs globaux, le curator poursuit les mêmes qu'un professionnel de l'info-doc : fournir à son public des informations de qualité, et en proposer un système d'archivage (la plate-forme de curation en l'occurrence) ; à long terme, devenir une référence sur un sujet précis (le nœud d'information d'un réseau pour le curator, d'une organisation pour le veilleur), démontrer son expertise pour la recherche d'informations appliquée à un domaine particulier.

De prime abord donc, la veille et la curation semblent s'accorder quant à leurs finalité et mode de fonctionnement : collecter, sélectionner, trier, archiver et diffuser l'information. Cependant, curation et veille ne sont pas équivalents mais en quoi se distinguent-ils ?

\section{L’accomplissement de soi comme première motivation}

La première différence notable est liée à la motivation. En effet, la curation n'est pas une activité salariée : elle ne répond pas à des contraintes organisationnelles et, surtout, n'a pas de commanditaire identifié. Le fait de sélectionner, ré-agencer et diffuser du contenu peut répondre à un besoin personnel information (tout comme l'utilisation des plates-formes de bookmarking), ou encore à un certain désir égotique de mise en valeur de soi et de ses connaissances d'un outil ou d'un sujet. Parfois, il s'agit d'alimenter une communauté thématique en information. Dans tous les cas, ce qui motive un curator n'est pas l'accomplissement d'une mission définie, mais bien l'accomplissement de soi. Il n'est pas ici question d'amateurisme d'une pratique par rapport à l'autre (de nombreux veilleurs font par exemple de la curation pour leur propre compte), mais bien de souligner qu'une erreur dans le choix d'une information n'a pas

1 http://forrester.typepad.com/groundswell/2010/01/conversationalists-get-onto-theladder.html

2 User Generated Contents, technologies habituellement associées au phénomène « 2.0 » 


\begin{tabular}{|c|c|c|}
\hline \multicolumn{3}{|c|}{ Quels apports du veilleur/documentaliste aux pratiques de curation de ses collaborateurs ? } \\
\hline Pratiques & Curateur & Veilleur \\
\hline Collecte & $\begin{array}{l}\text { Collecte libre en fonction } \\
\text { des centres d'intérêts }\end{array}$ & Structuration de la collecte, apports d'outils et de méthodologies \\
\hline Archivage & Pas d'archivage ou indexation libre & Proposition d'outils, mise en conformité avec le système interne \\
\hline Choix des sources & Choix libre en fonction des affinités & Proposition de critères de qualification des sources, vérification de la véracité des informations \\
\hline Diffusion & $\begin{array}{l}\text { Diffusion sur les outils propres } \\
\text { au curator, contexte mouvant }\end{array}$ & Contextualisation des informations, ciblage des destinataires, structuration de la diffusion \\
\hline Analyse & Rare analyse des informations & $\begin{array}{c}\text { Insertion des informations collectées par le curator dans un contexte d'analyse stratégique propre } \\
\text { à l'entreprise }\end{array}$ \\
\hline
\end{tabular}

le même impact pour le veilleur et son organisation que pour le curator.

Autre différence : les objectifs. La veille vise à répondre à des besoins identifiés, et à appuyer la prise de décision. Pour cela, la mise en place d'une stratégie de veille passe par un dialogue avec ses commanditaires, par la prise en compte du fonctionnement global de l'organisation, ou encore par l'évaluation des moyens à disposition. La curation, elle, ne répond pas à des objectifs précis (hormis ceux liés à la motivation du curator) et ne vise pas explicitement à faciliter la prise de décision. Pour schématiser, la pertinence des informations collectées par le curator dépend du public auquel il s'adresse (chacun faisant son choix) et de sa propre vision du sujet qu'il traite ; un veilleur définit la pertinence des informations qu'il sélectionne en fonction des objectifs de ses commanditaires et des décisions/actions qui en découleront par la suite.

Pour répondre de manière régulière et fiable à ses objectifs, un veilleur doit se reposer sur une méthodologie établie et qui soit adaptée quelles que soient les demandes de ses "clients » et la thématique concernée. Cette méthodologie est fondée sur le choix d'outils qui doivent s'accorder au mieux aux besoins exprimés par le commanditaire (sur la typologie des contenus à collecter par exemple) et à ceux du veilleur. Pour le curator, le choix de l'outil s'opère généralement non pas par sa pertinence dans l'absolu ou pour un besoin identifié mais par sa facilité et sa souplesse d'utilisation

Ensuite, la sélection des sources d'informations, dans une stratégie de veille, s'effectue en fonction de critères réfléchis : qualification des auteurs et informations diffusées, expertise et autorité de la source, etc. Pour la curation, cette sélection ne s'applique pas de la même manière : tout comme pour l'utilisation d'un agrégateur à des fins personnelles, le curator choisit ses sources à partir de critères qui lui sont propres (affinité, ton de l'auteur, types de contenus par exemple), voire sans critères explicites du tout. Enfin, et toujours d'un point de vue méthodologique, la veille passe par l'analyse des informations collectées alors que la curation peut parfois ne s'appuyer que sur une forme de prescription par d'autres curators (une reprise immédiate facilitée par la multiplication des boutons de partage, par exemple), avec le risque constant de (re)diffuser des informations douteuses ou erronées.
La nature (ou le type) des outils sur lesquels s'appuient curation ou veille implique également des différences dans les pratiques. Les usages du curator sont généralement induits par les outils qu'il utilise. Si, lorsque l'on observe des actions de curation, le choix des outils de collecte est difficilement décelable, celui des outils de diffusion (les fameuses platesformes de curation, ou les UGC de manière générale) en revanche est facilement identifiable. Cette différence est de taille car là où la curation est libre dans ses modes de diffusion, la veille est contrainte par les modes de consommation informationnelle des commanditaires et des publics dans l'organisation. Or, dans la veille, la diffusion est l'un des facteurs clés de réussite : pourquoi collecter de l'information si personne ne la lit?

\section{La différence dans les usages}

La diffusion opérée par un curator varie quant à elle en fonction du public qu'il vise et donc de la plate-forme qu'utilise ce dernier, des interconnexions possibles entre ses différents comptes, ou encore des capacités de réagencement (ajout de métadonnées, de commentaires, etc.) que la solution permet. Le curator a le choix des informations qu'il met en avant (ou se reposera sur l'algorithme de certains outils) là où le veilleur reste dépendant des besoins de son client (voire de certaines restrictions techniques dans son organisation).

En somme, ce qui différencie la veille et la curation sont les usages qui en découlent : structurés et motivés par des stratégies organisationnelles pour la veille, au gré des besoins et induits par les technologies utilisées pour la curation.

Le professionnel de l'info-doc, s'il n'a donc pas à s' « inquiéter $»$ des pratiques de curation en terme de concurrence, peut néanmoins s'appuyer sur celles-ci. Tout d'abord en interne, en identifiant les potentiels curators et en leur apportant un soutien méthodologique ou d'analyse de leur veille (si celle-ci répond à certains objectifs). Et, bien entendu, en s'appuyant sur leurs résultats s'ils consentent à les partager. Ensuite, en percevant les curators comme des «filtres humains » dont la sélection de l'information permet de repérer les sources qui construisent les connaissances sur un sujet d'une communauté ou d'un réseau, voire de déceler de potentiels signaux faibles... ou forts, le fait d'être dans la tendance (et donc de la suivre en diffusant des informations ad hoc) étant une manière d'être plus aisément reconnu et identifié sur le web.

Pour conclure, la curation et les plates-formes qui lui sont associées deviennent des outils pour les veilleurs. Si la curation se détourne de la veille dans la pratique, elle n'en reste pas moins une forme de sélection de l'information à intégrer dans un processus de gestion de l'information. • 
[ IST ] La curation scientifique apparaît aujourd'hui sous un double aspect : numérique et social. La première permet de gérer les données de la recherche tout au long de leur cycle de vie alors que la seconde, en s'appuyant sur les outils grand-public, offre aux chercheurs de nouvelles opportunités de diffusion de sa veille. Une dualité féconde.

\section{Les deux faces de la curation scientifique}

La curation numérique (Digital Curation), fortement associée à l'e-science, s'occupe de maintenir, préserver, valoriser les données numériques de la recherche à travers leur cycle de vie. La curation sociale (individuelle et collective) permet à l'individu-chercheur d'éditorialiser des contenus issus du Web et de donner à lire sa représentation d'une thématique, et au collectif-chercheurs soit de lire les représentations de recherche sociale, soit d'agir collectivement dans le cadre d'une même activité (crowdsourcing, open peer review, etc.)

La racine latine curatio désigne le fait de s'occuper de, d'effectuer un soin. Le curator signifie conservateur dans son acception anglaise. Ainsi, la définition de curation pourrait correspondre à celle de la conservation : il s'agit de gérer des collections (colliger, sélectionner, restaurer). Si la science a toujours fait cela, certains domaines scientifiques ont vu croître les données expérimentales et d'observation de manière très importante avec l'évolution des outils « d'acquisition» (par exemple, les séquenceurs pour la génomique, de nouvelles sondes ou nouveaux télescopes pour l'astrophysique). Ce phénomène de « déluge de données $»^{1}$, contenu par les systèmes

\footnotetext{
1 Les articles suivants révèlent le début de l'intérêt du sujet à la fin des années 80-début 90 : R. Wolkomir, « NASA's data deluge ». Air and Space, 1989, 4, p. 78-82 et P. Aldhous, « Managing the genome data deluge ». Science, 1993, 262(5133), p. 502-503.

2 A.J.G. Hey, S. Tansley et K.M. Tolle. The fourth paradigm: dataintensive scientific discovery. Microsoft research Redmond, 2009 3 La Science, l'ensemble des connaissances scientifiques

4 Digital Curation Centre . http://www.dcc.ac.uk/

5 Centre informatique national de l'enseignement supérieur : http://www.cines.fr
}

de gestion de base de données (SGBD), n'a cessé d'évoluer et a logiquement débouché sur la question du partage des ressources. Si l'enregistrement des données peut être normalisé en amont par discipline ou domaine scientifique (standard ISO, descripteurs minimum, classifications dédiées, etc.), l'automatisation de processus de collecte et l'imposante quantité d'information manipulée sont des facteurs d'inévitables erreurs (à la source ou à l'enregistrement). La curation numérique prend alors tout son sens en travaillant sur la qualité des données et en les rendant pérennes.

\section{Un domaine de recherche à part entière}

Jim Gray $^{2}$ met en avant le passage des méthodes empiriques, débutées il y a quelques millénaires, aux méthodes d'abstractions théoriques démarrées il y a quelques siècles, puis aux méthodes de traitement informatique des données initiées il y a quelques décennies pour enfin aborder l'exploration des données à travers les cyber-infrastructures. Ce dernier paradigme ne peut exister sans curation, c'est-à-dire sans travail de sélection et de nettoyage de l'information ouvrant pour la qualité de données durablement stockées. La curation permet une recherche transdisciplinaire et autorise la découverte de connaissances (Knowledge Discovery in Databases, KDD). Il s'agira alors de traquer les enregistrements mal renseignés, invalides ou erronés et de maintenir l'interopérabilité des bases de données en gérant des ontologies et des protocoles de communication électronique. Il conviendra encore de désherber ou du moins gérer le flux des données dans le temps et de rendre accessible ce travail avec des outils de datamining ou de visualisation de données servant à la fois le travail de recherche d'information, l'épistémè et la curation par les experts.

Mais la curation se professionnalise également et s'octroie ainsi le statut de domaine de recherche à par entière. Le Digital Curation Centre (DCC) ${ }^{4}$, financé par le JISC, est une structure $/ / / / / / /$ 
/////// d'expertise pour la curation des données en GrandeBretagne. Elle ne semble pas très éloignée de notre $\mathrm{CINES}^{5}$, mais ce dernier met essentiellement l'accent sur l'archivage pérenne bien qu'il ait de nombreux projets de curation avec des partenaires scientifiques. La revue International Journal of Digital Curation ${ }^{6}$, créée en 2006, est un des supports de la recherche en curation. L'éditorial du premier numéro indique bien l'enjeu de ce domaine: «La curation numérique apparaît aujourd'hui comme consubstantielle du défi intellectuel et des actions collaboratives pour un large éventail d'individus, dont les chercheurs, les informaticiens, les bibliothécaires, les archivistes, les décideurs politiques et les partenaires financiers de la recherche ${ }^{7}$. Les projets d'e-science, de cyber-infrastructure, d'open data scientifique vont donc de pair avec une curation numérique comme l'empirie des premières sciences avait besoin de curation.

\section{Curation sociale \\ et surcharge informationnelle}

Même si des outils peuvent nous aider, nous subissons une surcharge informationnelle sur le Web (information overload) et l'activité du chercheur sur le réseau n'échappe pas à la règle. Les outils du Web 2.0, qui ont transformé l'internaute en producteur/ diffuseur d'informations, et dans le même temps, en suiveur de « fils » d'information, ont leur déclinaison scientifique.

de nouvelles relations. Certains réseaux partent du groupe pour partager des informations, d'autres s'étayent sur les informations partagées pour construire des communautés ${ }^{9}$.

Grâce à ces outils orientés "sciences », le chercheur opère une forme de curation, dans la mesure où il va mémoriser, publier et suivre certaines informations et pas d'autres. Si on leur ajoute les outils génériques et dédiés à la sélection comme Scoop.it, Paper.li, Storify, Curated.by, l'internaute-chercheur possède de quoi éditorialiser «ses » contenus et jouer le gatekeeper $^{10}$ de " son domaine ", bref proposer une curation sociale. Comme l'écrit Dominique Cardon, « faire des internautes des curateurs, c'est d'abord tirer les conséquences de deux transformations importantes des pratiques du Web : le développement de la recherche sociale (en opposition à la recherche lexicale ou sémantique) et l'accroissement de la critique du pouvoir des gate-keepers sur l'agenda de l'information. $»^{11}$.

L'ensemble des curations peut-il servir de ressources fiables pour découvrir ou éclairer des résultats ? Les fils d'information produits sont-ils pérennes ou ne sont-ils que temporaires ? Quels sont les enjeux de ces outils commerciaux utilisés à titre gratuit ? Il semble donc que cette curation sociale vaut d'abord pour l'individu-chercheur et qu'elle sert la communauté scientifique comme flux et comme happening balayant les catégories et les institutions.

\section{Curation et indicateurs}

Toutefois, on peut relever plusieurs types de curation sociale collective. Le crowdsourcing consiste à faire participer les internautes à l'identification d'éléments publiés sur le Web. L'expérience d'une collection d'images déposée sur Flickr par la Bibliothèque du Congrès américain montre l'efficacité de ce type de curation. L'open peer review permet à tous, mais d'abord aux spécialistes du domaine, de commenter un texte soumis à publication. L'expérience \#arseniclife montre ainsi comment des tweets et des billets de blog ont permis la non-publica-

Sous ce vocable 2.0 sont rassemblés les platesformes de partage de références bibliographiques ou webographiques (par exemple CiteULike, Delicious, Diigo, Zotero, Mendeley), les weblogs et les platesformes de réseau social à caractère scientifique. Les premières sont intéressantes à observer en ce qu'elles s'apparentent au Memex ${ }^{8}$ et aux bases de données bibliographiques. Elles mémorisent et organisent les références, elles les lient, permettent leur mise en commun et donc un travail collaboratif. Les weblogs représentent la version « en ligne » des carnets de recherche. Ils offrent à lire les traces du cheminement scientifique, la mémorisation du travail de terrain. Du site de chercheur aux « agrégateurs » de billets (Postgenomic) en passant par les plates-formes dédiées à la recherche (Hypotheses.org), les blogs de chercheurs occupent désormais une place importante dans l'agora scientifique. Les plates-formes de réseau social comme ResarchGate ou SciLink agrègent un certain nombre d'informations liées à chacun des membres du réseau (bibliographies, textes, appartenance à un laboratoire ou université) et impriment tion d'un texte scientifiquement peu étayé dans la revue Science ${ }^{12}$. Ce type de curation s'érige même en indicateur sous l'appellation de Retraction Index $x^{13}$.

6 IJDC : http://www.ijdc.net/index.php/ijdc

7 C. Rusbridge et L. Lyon, « Editorial ». International Journal of Digital Curation, 2006, 1(1), p. 1-2. Traduction libre.

8 Le Memex est un ordinateur analogique fictif décrit par Vannevar Bush dans un article publié en 1945 dans The Atlantic Monthly.

9 G. Gallezot et 0. Le Deuff. « Chercheurs 2.0 ? » Les Cahiers du numérique, 2009, 5(2), p. $15-31$

10 Le «gardien du temple », « le passeur »

11 D. Cardon, "Tous éditeurs ? Les promesses incertaines de la "curation" », C/blog,

26 avril 2011, http://cblog.culture.fr/2011/04/26/tous-editeurs-les-promessesincertaines-de-la-\%C2\%AB\%C2\%A0curation\%C2\%A0\%C2\%BB

12 C. Zimmer, «This Paper Should Not Have Been Published », Slate, 7 déc. 2010, http://www.slate.com/articles/health_and_science/science/2010/12/this_paper should not have been published.html et « The Discovery of Arsenic-Based Twitter. How \#arseniclife changed science », Slate, 27 mai 2011,

http://www.slate.com/articles/health_and_science/science/2011/05/the_discovery of_arsenicbased_twitter.html

$13 \bar{F}$. C. Fang et A. Casadevall, « Retracted Science and the Retraction Index ». Infection and Immunity, 2011, 79(10), p. 3855-3859. 


\section{témoignage}

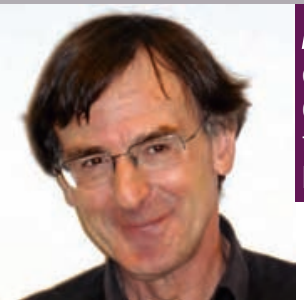

Alexundre Serres est maître de conférences en sciences de l'information et de la communication à l'Université Rennes 2 et co-responsable de l'Urfist de Bretagne-Pays de la Loire. Ses recherches actuelles portent sur la culture informationnelle, la formation documentaire des étudiants et l'évaluation de l'information sur Internet, sur laquelle doit paraître, aux éditions (\&F, un ouvrage intitulé Dans le labyrinthe.

alexandre.serres@uhb.fr

\section{Les outils de curation nous obligent à} réinventer des mécanismes de confiance

Qu'est-ce que la curation modifie quant à la valeur d'une information?

Tout dépend de ce que l'on entend par « valeur de l'information ». Si l'on entend sa crédibilité (la croyance qu'on peut avoir quant à sa validité), la curation peut apporter certaines garanties, du moins quant à la crédibilité du médiateur, voire de la source sélectionnée par ce médiateur. Mais la crédibilité du curateur ne garantit pas obligatoirement celle de l'information elle-même! Si on entend par valeur de l'information sa validité interne, i.e. la qualité de son contenu, sa cohérence, sa nouveauté, etc., alors la curation, réalisée par des experts, des professionnels de l'information ou tout simplement des internautes compétents et rigoureux, permet une diffusion sélective d'informations de qualité. Mais comme le dit Daniel Bougnoux ${ }^{1}$, « la valeur principale d'une information réside dans sa pertinence ». Ce qui « fait information » pour l'un ne le fera pas pour l'autre et la valeur d'une information, au sens de son impact et de sa pertinence, dépend avant tout de sa réception par une personne précise, qui lui donnera sens dans un contexte et pour des besoins précis. Et, de ce point de vue, la curation, comme n'importe quel média, ne change strictement rien! Le mode d'accès à une information (via une plateforme de curation, un moteur de recherche ou tout autre moyen) n'a pas vraiment de conséquence directe sur la valeur de celle-ci. L'information n'existe pas « en soi », elle est toujours construite et c'est un regard humain spécifique qui lui donne, ou non, une valeur.

Linformation peut-elle valoir plus, finalement, que la source dont elle provient ?

Jusqu'à quel point la valeur d'une information estelle liée à sa source ou, dans le cas de la curation, à son médiateur? Car les curateurs ne sont pas à la source des informations, ils n'en sont que les médiateurs, les diffuseurs. Source et information ne se recouvrent pas : une source jugée crédible peut donner une information fausse et, à l'inverse, on peut trouver dans une source peu fiable des informations exactes. Linformation, dans son contenu, dans sa « plausibilité intrinsèque », est en grande partie indépendante de sa source, et donc encore plus de ses médiateurs. Le récepteur décide, in fine, de la valeur de l'information. Il n'en reste pas moins que le curateur qui a sélectionné et éditorialisé une information dans le flot continu du Web change, non pas la valeur initiale, mais le statut, l'impact social et la visibilité de cette information. La curation est, à ce titre, peut-être plus une activité de communication que d'information. Une plateforme de curation donne à voir, met en lumière des informations choisies par quelqu'un.

Comment valider une source « humaine " particulièrement par rapport à une source plus classique?

Tout est question de confiance et de crédibilité. En qui avoir confiance sur les réseaux et sur Twitter? Aucune recette ici, mais une combinaison entre d'une part la qualité du réseau social d'appartenance, de la communauté, et d'autre part l'expérience personnelle, l'observation, la vigilance et l'esprit critique. Plus qu'ailleurs, la confiance procède par délégation sur les réseaux sociaux et sur Twitter : si Untel est suivi par quelqu'un en qui j'ai confiance, alors j'aurai tendance à lui accorder ma confiance, etc. Les outils de curation, de même que Twitter et les réseaux sociaux, nous obligent à réinventer des mécanismes de confiance, condition première de la « validation » d'une source humaine. Cette confiance est toujours un pari, elle est relative, fragile et certainement pas soluble dans les algorithmes ! Aucun outil ne peut, seul, valider la qualité, l'authenticité et encore moins la véracité d'un tweet. Et de son côté, si le collectif peut représenter une garantie forte de validation d'une information ou d'une source, il ne saurait dispenser l'individu de sa propre vigilance personnelle. •

Propos recueillis par Christophe Deschamps.

1 Daniel Bougnoux, Introduction aux sciences de la communication, Éditions La Découverte, 1998, p. 79 


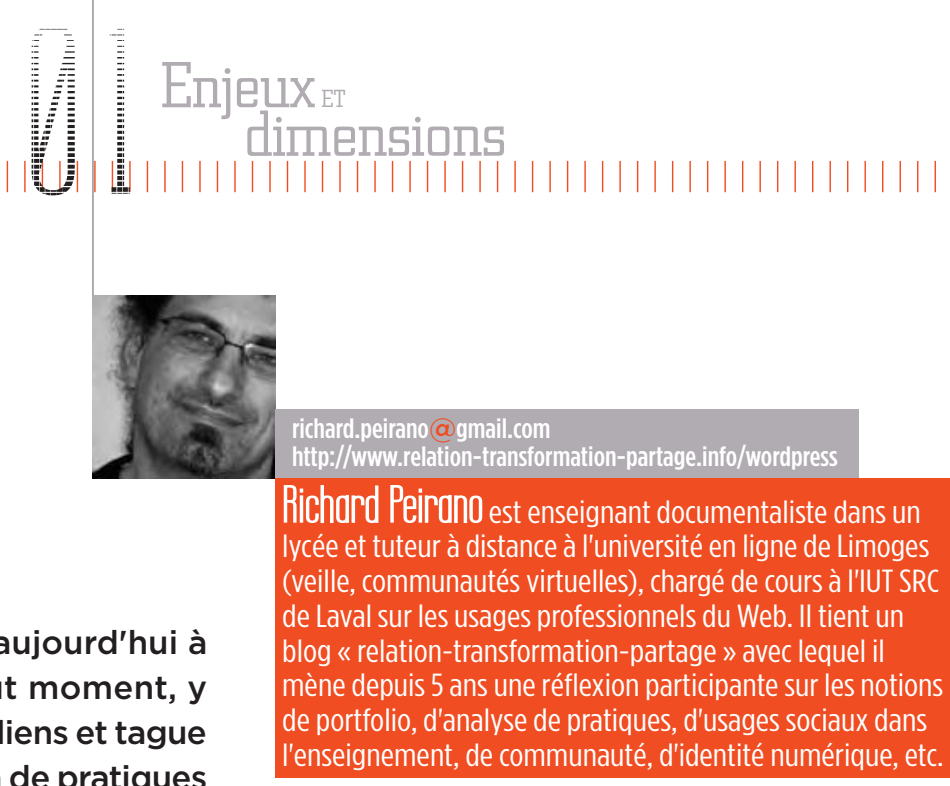

des contenus sur Facebook. Il s'agit déjà de pratiques

de curation. L'enseignant, alors, peut s'appuyer sur

les pratiques existantes des élèves afin de proposer

des pratiques scolaires conformes et efficaces qui

déboucheront sur des pratiques sociales de référence.

\section{La curation à l'école,}

\section{vers une culture de l'information}

Que veut dire curation dans l'environnement scolaire? Cette question interroge le rôle de l'outil dans l'apprentissage de l'élève ainsi que son inscription dans le dispositif d'enseignement voulu par l'enseignant. L'outil en lui-même et le monde numérique dans lequel il s'insère renvoient également, il me semble, à de nouveaux apprentissages qui seraient comme une grammaire du Web. Car, au final, il s'agit de faire de l'élève cet honnête homme, à la culture affirmée en tant que lecteur et producteur d'informations, capable d'évoluer dans cet environnement numérique.

\section{Les conditions d'une rencontre}

La curation consiste à surveiller, lire, commenter, re-publier et partager du contenu. La nouveauté réside donc dans l'outillage de la démarche et dans la gamme d'outils choisie et non dans la démarche en elle-même, déjà présente, par exemple, dans la glose médiévale... et à l'école.

Les outils de curation sont adossés à des pratiques numériques installées comme le partage de liens ou l'éditorialisation de contenu qu'ils vont, pour certains, automatiser. Ils s'appuient sur Twitter, au centre de cette économie du lien ${ }^{1}$. La rencontre avec l'école est donc envisageable si on considère la curation comme un outillage de l'apprentissage de l'élève qui va lui permettre de:

- mettre en forme sa rencontre avec un texte ${ }^{2}$ afin de produire un contenu sur le contenu ;
- apprendre par confrontation entre ce qu'il sait et ce qu'il lit ;

- construire cet apprentissage en se confrontant aux autres dans sa communauté d'apprentissage ;

- expliciter ses propres connaissances qui seront un jalon sur lequel continuer à apprendre.

L'enseignant, alors, apporte moins le savoir que la mise en place de dispositifs d'enseignement où l'élève va interagir, seul ou en groupe, sur des textes fournis ou qu'il doit trouver, avec des outils imposés.

\section{Outiller la démarche d'apprentissage}

Voici des exemples qui proposent quelques pratiques possibles de cet outillage, autour de Twitter, Diigo, Tumblr et Pearltrees.

Laurence Juin ${ }^{3}$ est l'une des premières à rendre visibles des activités pédagogiques avec Twitter. Elle le considère alors comme un outil d'écriture permettant de dynamiser le travail scolaire, individuel et de groupe. Au fil de l'expérience, il devient l'équivalent du stylo/page, à savoir un outil simple, adaptable à beaucoup de situations pédagogiques. C'est notamment la pratique du live-tweet qui consiste, à partir d'un hashtag ${ }^{4}$ défini a priori par l'enseignant, à faire prendre des notes aux élèves à partir d'un texte commun.

L'accumulation des tweets peut faire émerger des répétitions, soulignant ainsi l'importance d'un passage. Dans le même temps, un élève peut faire part de ses incompréhensions ou d'une idée que lui donne cette lecture. L'enseignant ou les pairs dans la classe interviennent alors pour engager la discussion, renforcer un propos, ou aider à résoudre un conflit de sens. 
Ce travail autour du lien est aussi possible avec un outil de bookmarking social comme Diigo. Lors d'une recherche en ECJS ${ }^{5}$ terminale, six groupes d'élèves doivent travailler sur un thème ${ }^{6}$. Diigo va permettre de conserver des liens, de les travailler (surlignage/annotation) et de produire des métadonnées (résumé/tags). Puis chaque élève va signaler les liens trouvés à ses camarades via des groupes Diigo privés, dans lesquels ils vont pouvoir commenter les trouvailles de chacun et débattre ensemble autour de la ressource.

D'autres outils pourraient permettre ce travail comme par exemple Tumblr, outil de microblogging, qui donne la possibilité à l'élève de bloguer sur un lien, c'est à dire le confronter à ses connaissances afin de les transformer dans un premier temps et de tester sa compréhension auprès des pairs du groupe.

Une autre activité envisageable s'appuie sur le travail d'une collègue enseignante documentaliste ${ }^{7}$. Lors d'une recherche, l'élève n'a souvent pas d'idées sur le sujet. Il s'agit alors dans un premier temps de lui faire acquérir une connaissance de base, via un document de collecte. On peut envisager de proposer à un groupe d'élèves, via un Pearltree ${ }^{8}$, une collection de quelques liens sur un sujet qu'ils vont lire, annoter, commenter, en extraire les mots importants et proposer un billet de blog, intégré ensuite dans le Pearltree. Le groupe peut alors commencer ses recherches pour enrichir cette connaissance de base et conserver les liens trouvés et annotés. L'intérêt du Pearltree réside ensuite dans la négociation en groupe de l'organisation des liens, via l'introduction de sous-pearltrees faisant office de rubriques. On profite alors de toute la souplesse du mindmapping pour classer les textes et les idées qu'ils contiennent.

Les outils de curation à l'école permettent, à partir d'un texte, un échange entre ce que je lis et ce que je sais, et entre ce que je lis et ce que les autres membres du groupe avec qui je travaille lisent et savent. Nous sommes là dans la définition de la communauté d'apprentissage, définie par Garisson ${ }^{9}$ comme « un groupe d'individus qui sont engagés de manière collaborative dans un échange et une réflexion déterminés et critiques, pour construire le sens et confirmer la compréhension mutuelle. »

\section{Acquérir une culture de I'outil}

Derrière cette logique de l'outillage, il y aussi l'idée $\mathrm{du}$ forgeron qui ne peut apprendre qu'en forgeant. Nous sommes confrontés aujourd'hui à de jeunes usagers numériques qui possèdent une culture technique assez pauvre, aussi bien en terme de représentation de ce qu'est un outil numérique qu'en terme de connaissance de la gamme des outils à disposition.

Les séances présentées ci-dessus accroissent la perception de la diversité des outils et des usages et les inscrivent dans une perspective d'apprentissage et non plus seulement récréative. Elles trouvent leur légitimité en s'appuyant sur les pratiques numériques en même temps qu'elles épousent une démarche d'apprentissage dans un dispositif pédagogique qui a du sens.

Mais l'usage de ces outils n'est pas neutre. Ils sont pilotés par des modèles marchands, organisés autour des traces laissées par les usagers, ici des élèves. L'enseignant doit être au clair avec ses valeurs, ses intentions, ses objectifs ${ }^{10}$. La question de la mise en forme et de la facilité peut aussi être avancée. Par exemple, pourquoi l'élève va-t-il réfléchir à la maquette d'un journal quand des outils en ligne proposent des gabarits prêts à l'emploi pour une création honnête à moindre coût et à moindre effort ${ }^{11}$ ?

\section{Acquérir une grammaire}

Pour dépasser ces débats, il convient de faire de l'outil un objet d'apprentissage en soi et de passer à l'acquisition d'une grammaire numérique. Nous n'allons pas apprendre aux élèves à se mouvoir dans l'univers numérique, nous allons leur en apprendre la grammaire et il nous semble que c'est à l'enseignant-documentaliste qu'incombe cette mission, cette tâche. À partir des invariants fonctionnels que l'on trouve sur tous ces outils, il faut travailler les notions de profil, de mise en relation, de média social, de partage de lien, de publication, d'auteur, de communauté, etc. Il faut enseigner les logiques technologiques, industrielles, commerciales, juridiques, formelles et politiques au coeur de ces outils.

Avec l'ensemble de la communauté éducative, il faut combattre quelques idées reçues comme la facilité d'usage au détriment du sens, la gratuité comme un dû et non comme une forme économique contingente, la distraction au détriment de l'attention et du sens de l'effort, le copier-coller comme étape de l'apprentissage et non comme finalité... Et, au centre, il faut accompagner la présence en ligne des élèves, comme auteur numérique, citoyen, consommateur et futur salarié. Ce qui suppose de passer d'une pédagogie de la peur à une pédagogie de la présence.

1 Pierre Mongin. « 13 outils de curation selon vos besoins », 12 janvier 2012, Le mind mapping pour tous, http://www.mindmanagement.org/spip.php?article806 2 Terme que j'emploie indifféremment pour tout type de média.

3 Laurence Juin. « Un nouvel outil au service de ma pédagogie : Storify ! », 9 déc. 2011, Ma onzième année et les suivantes..

http://maonziemeannee.wordpress.com/2011/12/09/un-nouvel-outil-au-service-dema-pedagogie-storify

4 Mot-clé précédé d'un \# sur Twitter pour indiquer un thème traité ou une conférence suivie.

5 Éducation civique, juridique et sociale

6 Richard Peirano. «Sur Diigo : un scénario pédagogique en terminale », 2 oct. 2010, Relation, transformation, partage http://www.relation-transformation-partage.info/ wordpress/2010/10/02/sur-diigo-un-scenario-pedagogique-en-terminale 7 « Pourquoi (et comment) utiliser le document de collecte m'a permis d'abandonner la méthode de recherche par étapes », 8 décembre 2011, Mes docs de doc, http://mesdocsdedoc.over-blog.com/article-pourquoi-et-comment-j-utilise-ledocuement-de-collecte-dans-de-nombreuses-situations-de-recherche-91780637.htm 8 Outil de bookmarking qui se présente comme une arborescence graphique proche du mindmapping.

9 Alexandre Roberge. « Les éléments constitutifs d'une communauté d’apprentissage », 20 novembre 2011, Thot cursus, http://cursus.edu/dossiers-articles/articles/17789/leselements-constitutifs-une-communaute-apprentissage/

10 Laurence Juin. «Introduire les réseaux sociaux à l'école : prenons des risques ! (réfléchis) », ${ }^{\text {er }}$ septembre 2011, From Pennylane to..., https://fromplane.wordpress.com/2011/09/01/56

11 Richard Peirano. « Madmagz, Ceci n'est pas un journal », 18 décembre 2011, Relation, transformation, partage, http://www.relation-transformation-partage.info/wordpress /2011/12/18/madmagz-ceci-nest-pas-un-journal/ 


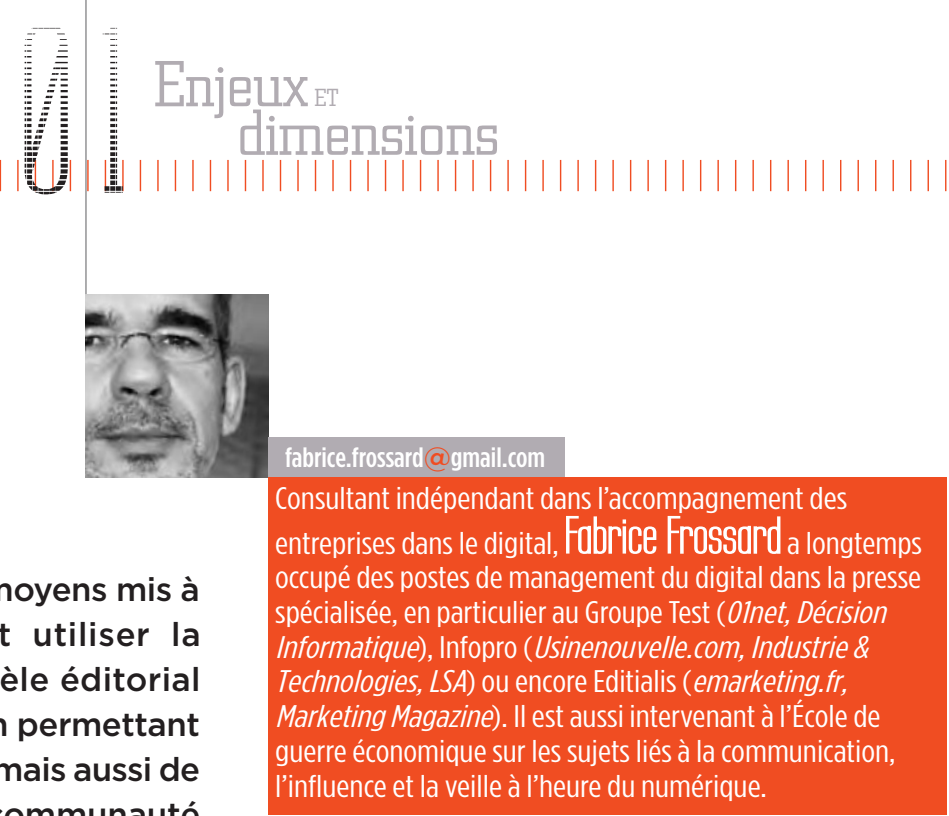

se constituer une organisation et une communauté fondées sur le partage social. Une pratique en devenir, mais aux risques avérés.

\section{Curation, presse et journalistes,}

\section{les liaisons dangereuses}

Le $1^{\text {er }}$ janvier 2012, Ben Smith est nommé rédacteur en chef de BuzzFeed, un site d'actualité qui se fonde en grande partie sur les informations issues des réseaux sociaux. Une anecdote? Pas vraiment. Ben Smith est un journaliste politique largement reconnu aux États-Unis. Après avoir été chroniqueur au NewYork Observer et au New-York Daily News, au blog Politicker, il devient l'une des vedettes de Politico.com, un site dirigé par deux anciens du Washington Post, John F. Harris et Jim VandeHei. Là, Ben Smith a su s'imposer comme un innovateur, tant dans ses analyses lors de la couverture des primaires démocrates en 2007 que dans l'approche des outils à disposition sur Internet pour la traiter. Mais, comme de nombreux journalistes, Ben Smith voit que son fil Twitter est plus fréquenté que son blog, que les informations livrées en temps réel sur les réseaux sociaux dissipent l'attention. Dont acte. En acceptant la rédaction en chef de BuzzFeed, Ben Smith devient le « primus inter pares» des curateurs de contenus et tente "la première vraie "social news organization", qui tient compte de la diffusion et du partage croissant de l'information par Twitter, Facebook et autres réseaux sociaux », comme il l'a déclaré au Niemanlab.org', un site dédié à l'évolution du journalisme.

Ce tournant opéré par Ben Smith est dans l'air du temps. Pour les journalistes et les éditeurs de médias, la curation est une question ancienne, aussi vieille qu'Internet, mais qui prend un nouveau tour avec l'explosion des médias sociaux, du temps réel et de l'inflation de données. Une question qui s'amplifiera encore avec l'extension des écrans d'accès.
Aujourd'hui, 90 \% des accès aux sites web d'infos sont encore issus des postes fixes, mais l'essor des Smartphones et autres tablettes, sans parler de celui de la télévision connectée, multiplie les points de contact avec l'information et les sollicitations de l'internaute. Dans ce contexte d'atomisation et de fragmentation de l'accès à l'information, le journalisme traditionnel bat de l'aile sous une double pression du temps et de l'hyper sollicitation, tandis que le lecteur ploie sous le joug de l'infobésité. La curation répond aussi à un défaut d'attention du lecteur. Comme l'écrivait très justement en 2008 Éric Scherrer alors prospectiviste pour l'AFP, « le trop plein d'informations, de stimulations et de choix, frôle la tyrannie. Et surtout, le temps est compté. L'autre grande rareté de ce nouveau paysage est bien aujourd'hui l'attention. » Submergé par l'information, les lecteurs disposent de peu de temps pour apprécier un article. Léconomie de l'attention est un des multiples enjeux auquel doit répondre la curation.

\section{Capter l'attention \\ en mettant en scène l'information}

Si nous revenons à Ben Smith, sa compréhension de la situation est évidente. En rejoignant BuzzFeed, son objectif est de retrouver cette attention en faisant évoluer la mise en scène de l'information, à la base de la curation. Le « curator » dans l'acception anglo-saxonne du terme est le responsable de la scénographie d'une exposition dans un musée, celui qui, par le choix et la disposition des œuvres, va donner une cohérence et un sens à l'ensemble. Transposée au journalisme, l'objectif de la curation est similaire. Dans un monde de flux, le journaliste se remet au centre du jeu en assurant ce rôle de filtre, de tri et de mise en scène et en sens de l'information. Une nécessité au vu de la production insensée de contenus sur la Toile. 
Sur le Web, la curation offre de nombreuses opportunités journalistiques. Aidé par des outils de veille et d'agrégation chaque jour plus nombreux (moteurs de recherche, Twitter, métamoteurs, interfaces de veille de type Feedly, Flipboard, etc.), le journaliste peut aisément réaliser un article, où se mêlent archives (vidéos, textes, photos), flux issus des réseaux sociaux et production éditoriale propre, destiné à apporter un éclairage nouveau sur un sujet donné et ainsi le valoriser par les enrichissements apportés. Un enrichissement qui se fera en deux temps. Le premier par le journaliste, le second par la communauté.

\section{Curation :}

\section{l'ultime avatar de la création}

\section{de contenus par l'utilisateur?}

Autre enjeu collatéral pour les journalistes, et surtout pour les médias, la constitution d'une communauté. La production d'information repose sur un double socle, celui du journaliste dans son rôle « de metteur en sens » et de la communauté, informelle dans un premier temps, pourvoyeuse d'informations non structurées via les blogs et médias sociaux et, dans un second temps, contributrice à l'enrichissement et à la distribution sur les réseaux de l'information mise en scène et structurée par le journaliste.

Pour les médias, quels qu'ils soient sur le Web, la curation doit en effet être l'opportunité de se constituer une communauté, fédérée autour d'un sujet commun, mais surtout doit permettre de se synchroniser avec elle dans une très ancienne dialectique: j'utilise des contenus issus de mon écosystème, je les ordonne et les enrichis et je les retransmets à chaque membre de ma communauté pour susciter une interaction : enrichissement, partage, « viralisation » ou « hyperdistribution». Pour ce faire, outre une curation intelligente, les nouveaux médias doivent aussi fournir un dispositif de « facilitation » du partage et de la prescription par l'utilisateur.

\section{Un nouveau modèle économique pour les médias}

Pour les médias, ce cycle vertueux autorisé par la curation redonne un ballon d'oxygène à un modèle économique à bout de souffle. C'est évident, mais il n'est pas inutile de le rappeler : la production de contenus journalistiques a un coût. La masse salariale représentée par une rédaction est l'épine dans le pied de tout éditeur de média. Inutile de revenir sans doute sur la descente aux enfers de la presse ces vingt dernières années. Les éditeurs, petits ou grands, sont tour à tour pris dans le cercle vicieux d'une chute d'audience progressive qui les conduit à réduire le nombre de journalistes, ce qui a pour conséquence de réduire la proposition éditoriale et donc, mécaniquement, le nombre de lecteurs et incidemment la surface publicitaire. Publicité qui représente en moyenne plus de $50 \%$ des revenus des titres de presse. Ce rapide aparté est nécessaire pour bien comprendre en quoi la curation offre à la fois de nouvelles opportunités éditoriales mais aussi un salut économique pour les éditeurs dont une partie substantielle des revenus se fera sur le numérique. De manière assez pragmatique, ces derniers voient dans la curation un moyen de produire et diffuser du contenu à faible coût, ou encore de couvrir des évènements importants sans mobiliser de nombreux journalistes. À ce titre, de nombreux exemples d'utilisation se font jour pour la couverture des élections présidentielle, américaine ou française. À l'instar du New York Times qui a mis en place une application mobile dédiée aux élections $2012^{2}$ dont une grande partie des contenus sera issue de sites tiers. En France, le site minutebuzz s'est allié avec le Nouvel Observateur pour lancer 2012.minutebuzz.com, consacré à l'actualité des Présidentielles 2012 vue depuis et par les réseaux sociaux, alimenté par un flux Twitter sélectionné par mots clés, mais aussi par la reprise d'articles, là encore, de sites tiers. Dans les deux cas, la curation de contenus et leur mise en scène, parfois minimale, n'apporte pas tant du sens qu'une couverture étendue de l'évènement en temps réel. Lagrégation du flux d'information par la reprise des informations issues des médias sociaux ou de la revue de presse prévaut sur la contextualisation, le sens et l'analyse.

Dans cette logique de masse critique et de flux, d'hyperdistribution de l'information, il y a de grands perdants. On l'a vu, la curation répond à plusieurs enjeux :

- Gestion de l'abondance

- Mise en scène de l'information

- Développement d'une communauté

- Hyper distribution de l'information

- Gestion de l'information en temps réel

- Réduction des coûts et des moyens

- Référencement de contenus et obtention d'une masse critique de mots clés

Néanmoins, pour vertueux qu'il soit, ce modèle ne résout qu'une partie des problématiques de la presse et, plus avant, des journalistes. Lévolution de leur métier est clairement liée dans l'environnement du Web à la rapidité de traitement de l'information sous la triple pression de l'abondance, du temps réel et de l'adhésion recherchée d'une communauté structurée ou non. Autant de variables avec lequel le journaliste doit jongler pour in fine accroître ou maintenir son audience. Toutefois, plusieurs questions restent en suspens, en particulier celle du temps de l'information. La fragmentation de l'audience, l'atomisation de l'information, l'accélération générale réduisent le nécessaire temps d'acquisition de l'information pour traiter un sujet de fond. Lenquête, la documentation, la réflexion : cette trilogie du journaliste d'investigation est battue en brèche par une théâtralisation sans doute excessive de l'information au détriment du fond. À voir si le mouvement de balancier entre ces deux pratiques, curation et investigation, pourra trouver un équilibre durable. Une nécessité pour notre métier. •

$1 \mathrm{http}: / / 0 w . l y / 90 M c k$

2 elections.nytimes.com/2012/mobile/app 


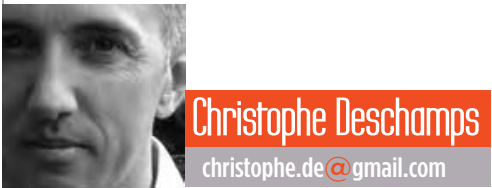

[ outils ] II est un signe qui ne trompe pas quant à la popularité d'un concept web innovant, c'est l'incroyable quantité de services qui s'en emparent dans l'espoir de s'y imposer. Si le devenir d'une idée se mesure à cela, alors nul doute que la curation a de beaux jours devant elle.

\section{Une offre foisonnante}

\section{pour des usages émergents}

Si le dynamisme d'un nouveau concept Web se mesure au nombre de services qui s'en réclament et à la diversité des modèles proposés pour le mettre en œuvre, alors le concept de curation en est un bel exemple. Un tableau comparatif mis en ligne par Pierre Tran ne recense pas moins d'une cinquantaine de services de curation'. Mais ce n'est rien en comparaison de la cartographie publiée par Robin Good qui répertorie à ce jour près de 200 services $^{2}$. Dans cet environnement hautement concurrentiel, il est essentiel de trouver un positionnement original permettant de se distinguer de ses adversaires. Le fait est que le concept de curation arrive dans un contexte sociotechnique particulièrement propice au foisonnement fonctionnel :

- la quantité d'informations à laquelle nous sommes soumis s'accroît d'année en année ; ainsi, une étude du Gartner Group indique que la quantité de données non-structurées va encore croître de 80 \% dans les cinq ans qui viennent ${ }^{3}$;

- la mobilité fait de plus en plus partie de notre quotidien ; les tablettes et smartphones nous permettent de consulter le Web en permanence et d'y partager des contenus au fil de l'eau ;

- les technologies de cloud computing se développent et donnent des gages de solidité et de sécurité qui amènent de plus en plus d'internautes, mais aussi de responsables informatiques, à leur faire confiance.
Résultat : les craintes d'utiliser des services en mode SaaS pour y déposer et partager des contenus en ligne s'affaiblissent.

Si le terme de curation est récent, les pratiques correspondantes sur le Web sont anciennes et passent par de nombreux outils. Plutôt que de nous concentrer sur les services dont le positionnement marketing est explicitement celui de la curation, nous avons donc choisi de « brasser large » en choisissant des services qui, a minima et sauf exception, répondent à la définition proposée par Wikipédia : « La curation de contenu [...] est une pratique qui consiste à sélectionner, éditorialiser et partager les contenus les plus pertinents du Web pour une requête ou un sujet donné ». Toutefois, cette définition oublie selon nous un élément essentiel. Si le fait de partager un flux thématique de découvertes et de montrer ainsi son expertise est une motivation forte pour le curateur, il n'en reste pas moins qu'il crée aussi ces collections pour son propre usage et utilise ces services pour leur capacité à archiver les éléments sélectionnés afin de les retrouver ultérieurement. Cette logique de personal knowledge management doit être prise en compte lorsqu'il s'agit d'évaluer des outils de curation.

\section{Comparatif des douze solutions de curation sélectionnées}

Le choix de ces services s'est effectué sur la base de tests formels ${ }^{5}$ ou non, réalisés par l'auteur au fil de l'eau depuis six ans. Cette connaissance préalable a été utilisée afin de sélectionner des outils qui ne sont pas des clones les uns des autres et d'envisager ainsi les différentes facettes du concept.

\begin{tabular}{|c|c|c|c|c|}
\hline & Positionnement & Tarif & URL & Divers \\
\hline Bagtheweb & curation & gratuit & www.bagtheweb.com & \\
\hline Delicious & social bookmarking et curation & gratuit & www.delicious.com & \\
\hline Diigo & social bookmarking & gratuit et une fonction Premium & www.diigo.com & \\
\hline Flipboard & magazine social & gratuit & www.flipboard.com & disponible uniquement pour iPad et iPhones \\
\hline Memolane & partage de sa vie numérique, storytelling & gratuit & www.memolane.com & \\
\hline Paper.li & curation & gratuit & www.paper.li & \\
\hline Pearltrees & curation collaborative & gratuit & www.pearltrees.com & \\
\hline Pinterest & curation & gratuit & www.pinterest.com & uniquement images et vidéos \\
\hline Scoop.it & curation & Gratuit, 2 versions payantes & www.scoop.it & \\
\hline Searcheeze & curation & gratuit & www.searcheeze.com & \\
\hline Searchteam & recherche d'information et curation sociale & $\begin{array}{l}\text { Gratuit, I version payante } \\
\text { (Zakta.com) }\end{array}$ & www.searchteam.com & \\
\hline Storify & curation & gratuit & www.storify.com & \\
\hline
\end{tabular}




\section{LES CRITĖRES D'ÉVALUATION DE L'OFFRE}

Trente-deux critères répartis en sept catégories ont été retenus pour évaluer les services de curation choisis.

Sources : comment le service de curation est-il alimenté en informations nouvelles susceptibles d'être diffusées?

- Externes : le curateur choisit les éléments à partager au fur et à mesure de sa navigation sur le Web.

- Internes manuelles : le curateur choisit d'utiliser certaines sources proposées par le service de curation. Il peut par exemple ajouter des flux RSS ou encore interroger un moteur de recherche généraliste (Google, Bing) à l'intérieur même du service et sélectionner les résultats à diffuser. - Internes via recommandations personnalisées : le curateur définit ses thèmes d'intérêt avec des mots-clés et le service lui propose des items issus de l'actualité (presse, blogs,...). II peut également s'agir d'un algorithme de recommandation basé sur l'historique de consultation/diffusion du curateur. - Intégration des réseaux sociaux : le curateur peut ajouter ses comptes de réseaux sociaux (Twitter, Facebook, Linkedln, etc.) au service de curation et rediffuser des items qui en sont issus. - Agrégateur de flux RSS : possibilité d'intégrer son propre agrégateur en ligne, généralement Google Reader, comme source.

Types de contenus intégrés : quels sont les types de contenus publiés par l'outil de curation?

- URL

- Images

- Vidéos

- Clipping web : il s'agit d'extraits de la page qui sont « découpés » par le curateur (images, textes)

- Fichiers bureautiques : .doc, .ppt, .pdf, etc.

Livrables proposés : quels types de livrables le service de curation permetil de diffuser?

- Page publique personnalisée : le curateur crée une page personnalisée et publique sur laquelle sont diffusés les éléments qu'il aura filtrés. - Diffusion manuelle vers les médias sociaux : le service se connecte aux médias sociaux du curateur et lui permet de diffuser manuellement les items sélectionnés.

- Diffusion automatisée vers les médias sociaux : le service se connecte aux médias sociaux et diffuse automatiquement les items que vous sélectionnez.

- Contenu embarqué (embedded) : le contenu peut être publié sur un blog ou un site web tiers via la génération d'un code à copier-coller.

- Email : possibilité d'envoyer un item par courriel.

- Création d'une newsletter : possibilité de générer une synthèse de la curation quotidienne et de la diffuser via courriel sous forme de newsletter.

- Flux RSS reprenant les items partagés.

- Optimisation du rendu pour smartphones, iPad, etc.

Optimisation pour l'usage personnel : - Lecture optimisée : le service se concentre sur l'amélioration de l'expérience de lecture des sources sélectionnées (sous forme d'un magazine par exemple).

- Utilisation mobile : I'interface de curation peut être utilisée sur smartphone, tablettes, iPhones, etc. (apps, widgets).

- Catégorisation par tags

- Autre système de catégorisation : le service propose un système de classement amélioré des éléments sélectionnés (ex : possibilité de classer par catégories, listes, groupes, bundles, etc.)

- Possibilité de conserver des éléments en privé (non publics)

\section{Aspect social et pratiques}

collaboratives :

- Réseau social : création de profils et fonctionnalités de mise en réseau (devenir « ami » avec, suivre Untel) - Possibilité de commenter les items publiés sur la page publique pour les lecteurs

- Possibilité de noter ou d'approuver un item (notes chiffrées, étoiles, « like », etc.)

- Curation collaborative : possibilité de créer des groupes et de mettre en œuvre une curation à plusieurs sur un même thème

- Curation collaborative privée : idem, avec la possibilité de créer des groupes privés

Éditorialisation :

- Possibilité de « pousser » un item en «Une»

- Personnalisation du thème de la page publique

- Possibilité pour le curateur d'ajouter des commentaires aux items sélectionnés

Moteur de recherche : présence d'un moteur permettant aux internautes de rechercher en plein-texte dans les contenus que vous avez enregistrés. •
Les services retenus et le positionnement qu'ils revendiquent sont les suivants (voir ci-contre) :

Si le foisonnement actuel des services de curation est la condition de leur richesse fonctionnelle, il crée de la confusion lorsqu'il s'agit de les classer dans une typologie. Les critères retenus ici permettent de sortir du positionnement marketing annoncé par les éditeurs en dressant une typologie qui tient compte des possibilités et fonctionnalités offertes par ces outils.

\section{Couverture des champs fonctionnels}

Le cumul des critères par catégorie fait apparaître les résultats suivants :

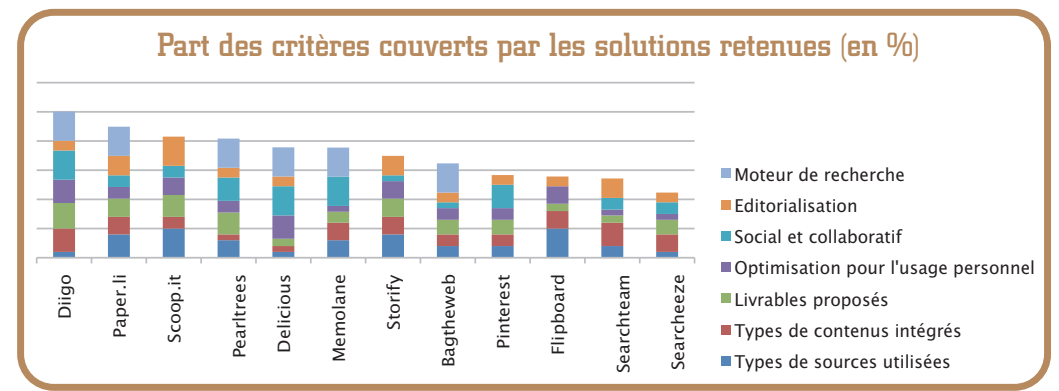

Étonnamment, c'est Diigo, un service positionné sur le social bookmarking depuis juillet 2006, qui arrive largement en tête, /////// 
/////// nous rappelant ainsi que le filtrage et la diffusion de contenus existaient bien avant qu'arrive le terme de curation. Pearltrees, qui se revendiquait initialement comme tel et Delicious, qui a initié le social bookmarking en 2003, sont d'ailleurs respectivement $4^{e}$ et $5^{e}$. Paper.li, présenté par ses créateurs comme service de curation mais souvent cité comme outil de publication automatique ${ }^{6}$, arrive en seconde position. Flipboard, qui se positionne comme un outil de veille informationnelle personnalisé, fait moins bien mais arrive tout de même avant deux services de curation. Les trois premiers services pure players sont donc Scoop.it $\left(3^{\mathrm{e}}\right)$, Pearltrees $\left(4^{\mathrm{e}}\right)$ et Storify $\left(7^{\mathrm{e}}\right)$.

\section{Popularité des critères pris en compte}

En moyenne, sur l'ensemble des services étudiés, aucune catégorie de critères n'est beaucoup plus développée qu'une autre.
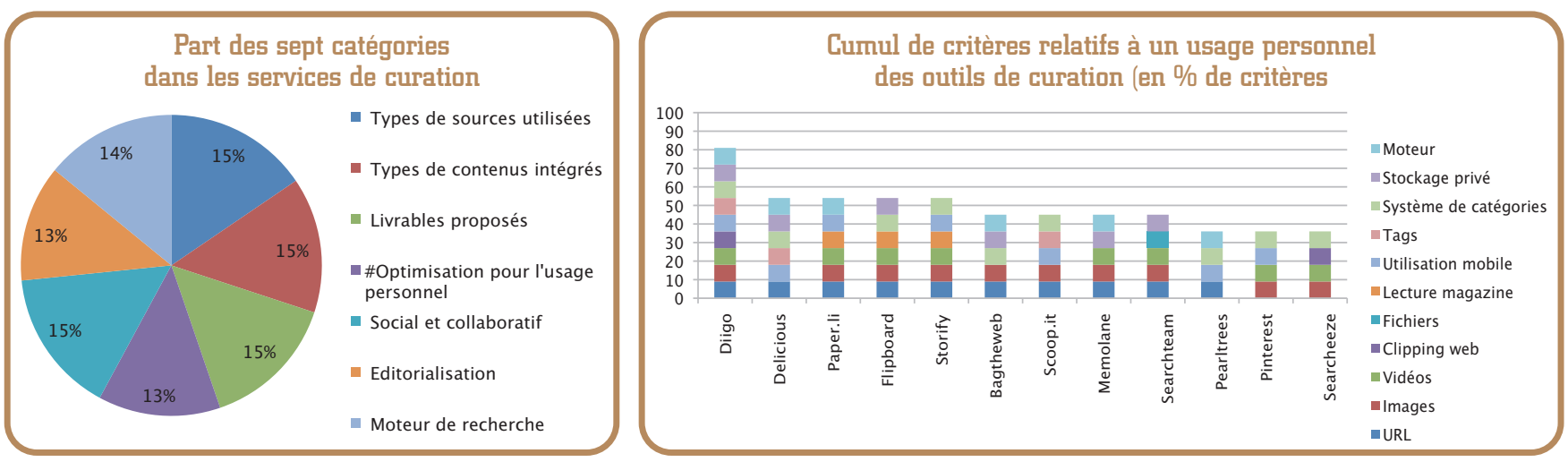

Ce classement basé sur une moyenne ne rend toutefois pas compte de la distribution des critères dans chacun des différents services. Sur ce point, les statistiques font émerger un premier groupe de trois catégories de critères relativement bien partagés : d'abord les types de contenus intégrés, puis les types de livrables susceptibles d'être diffusés, et enfin les fonctionnalités permettant de tirer parti individuellement de ces services qui, si elles ne sont pas très nombreuses ( $6^{\mathrm{e}}$ sur 7 catégories), sont en revanche distribuées de manière assez homogène parmi les outils étudiés.

Le second groupe comprend trois catégories de fonctionnalités dont le déploiement est plus irrégulier. On y trouve d'abord le type de sources proposées. Là où des services comme Delicious ou Diigo, traditionnellement orientés vers le social bookmarking, ne proposent que l'ajout manuel de contenus via une barre d'outils ou un bookmarklet, des services positionnés sur la curation, comme Scoop.it, ou sur la veille informationnelle personnalisée, comme Flipboard, intégrent flux RSS, réseaux sociaux personnels et recommandations personnalisées ou automatiques. Viennent ensuite les fonctionnalités d'éditorialisation, finalement pas ou peu présentes dans la majorité des cas sauf pour quelques outils comme Scoop.it, Paper.li et Storify. De fait, si Diigo est, de loin, le service couvrant le plus grand champ fonctionnel, il est peu présent sur ces deux catégories de fonctionnalités plus rares. Enfin, si les outils proposant un mode « réseau social » sont nombreux (9 sur 12), ceux proposant de mettre en œuvre une curation collaborative le sont moins ( 7 sur 12 ). Si en plus on souhaite qu'elle soit privée (dans une optique de veille collaborative par exemple), le choix se restreint alors à cinq outils.

\section{Les usages de la curation}

Afin de rendre ces positionnements plus concrets, nous avons choisi de confronter les services retenus à trois logiques d'usages possibles en sélectionnant pour chacune les critères les plus pertinents.

- Usage personnel et capitalisation

Ici, la curation est envisagée dans le cadre d'une veille informationnelle liée à une logique personnelle d'information et d'apprentissage. 


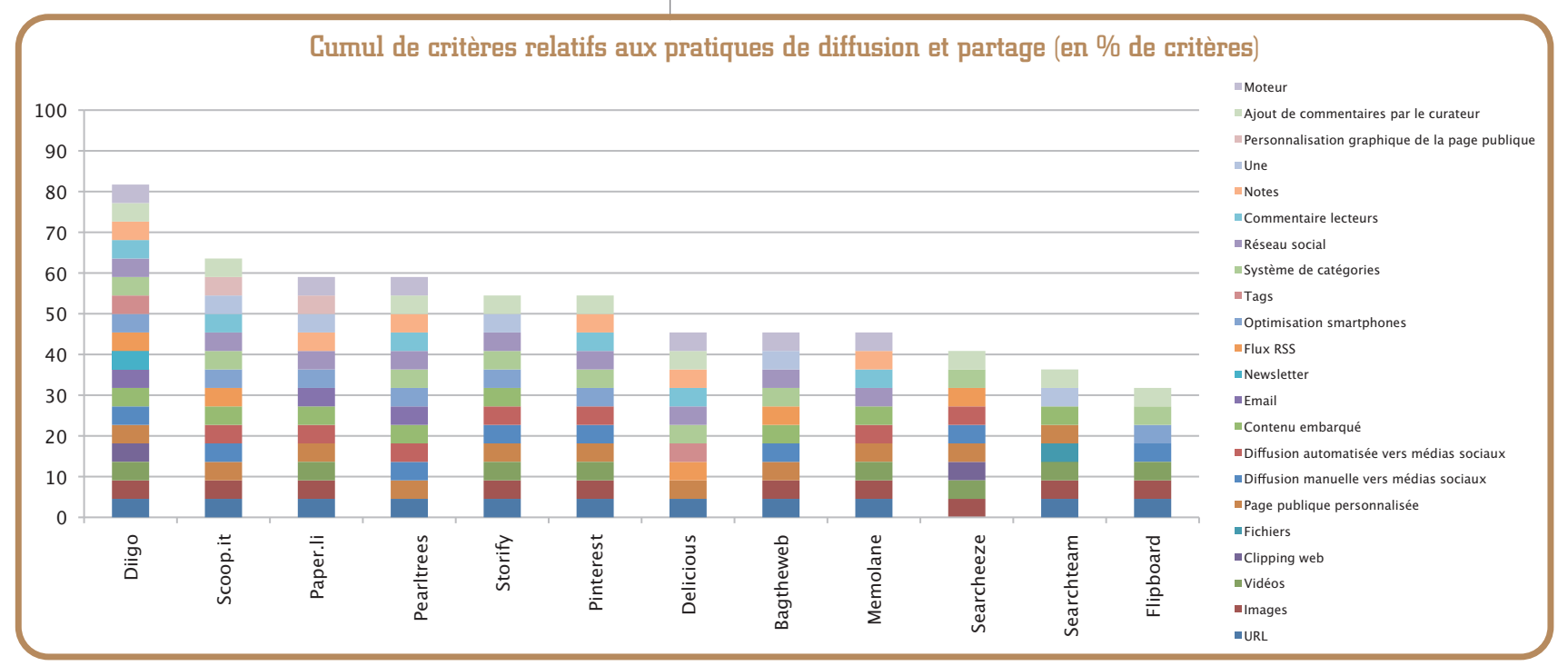

- Diffusion et partage

Il s'agit ici de fonctionnalités « classiques » de la curation telle qu'elle est définie couramment (voir cidessus).

De nouveau Diigo arrive en tête, mais les pure players se classent directement à sa suite. Sans surprise, Flipboard arrive ici en dernier.

- Curation collaborative

On peut avoir besoin de services permettant d'aller plus loin qu'une simple diffusion de contenus d'un individu vers plusieurs (one to many). Il peut s'agir par exemple de travailler sur une thématique à plusieurs et de créer une page publique collaborative (many to many), ou encore de mettre en place une veille collaborative n'ayant pas vocation à être diffusée. Il est nécessaire dans ce cas de disposer de services offrant un espace d'enregistrement accessible aux seuls membres d'un groupe.

De nouveau, Diigo et Delicious font la course en tête. Le premier service de curation est Pinterest qui ne permet toutefois que le partage d'images. Il faut descendre à la $5^{\mathrm{e}}$ position, avec Pearltrees, pour trouver un service de curation collaboratif (mais ne proposant pas de mode privé).

1 Pierre Tran, « Comparatif des plateformes de curation », http://socialcompare.com/fr/comparison/plates-formes-de-curationmd0synr.

Ce comparatif fait partie d'un article sur publié sur 01.Netle 16 mars 2011, http://pro.01net.com/editorial/529626/le-guide-de-la-curation(3)-les-outils/

2 Robin Good, "Best content curation tools",

http://www.mindmeister.com/fr/55395228/content-curation-tools-toaggregate-filter-edit-curate-and-distribute-any-type-of-contentupdated-weekly

3 Gartner Group, "Gartner identifies the top 10 strategic technologies for 2012", octobre 2011, http://www.gartner.com/it/page.jsp?id=1826214 4 Cf. Pierre Mongin, Xavier Delengaigne, Christophe Deschamps, Organisez vos données personnelles : l'essentiel du personal knowledge management. Dunod, 2011

5 Notamment pour la lettre Recherche \& Référencement édité par la société Abondance, http://recherche-referencement.abondance.com 6 Voir le classement de Pierre Tran cité ci-dessus.

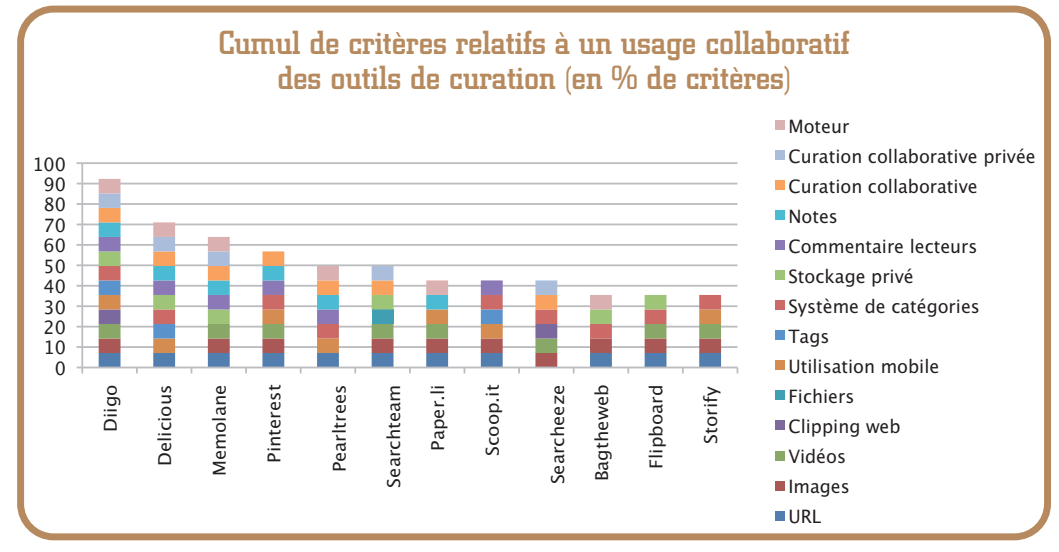

Ce comparatif rend compte avant tout de la couverture fonctionnelle de ces outils. Reste à le confronter à des besoins. En effet, si l'on souhaite partager avant tout des images et des vidéos, seul Pinterest est ici vraiment pertinent. Si l'on est très mobile, il faudra choisir un service optimisé pour le système d'exploitation de son smartphone ou de sa tablette (Storify, Scoop.it, Pearltrees ou Flipboard). Les critères proposés ci-dessus doivent donc être pondérés en fonction des priorités de l'utilisateur. Autre élément non pris en compte ici, l'expérience utilisateur. Certains services particulièrement bien conçus ont un potentiel d'addiction très fort. C'est le cas de Flipboard qui, de par la fluidité de son mode magazine, s'impose rapidement comme outil d'accès à l'information, compensant de fait des fonctionnalités de curation présentes mais limitées.

On le voit, les frontières sont encore floues et les jeux ne sont pas faits. Les services de curation se multiplient et, si leur usage dans une logique informationnelle est évident, les choix à effectuer doivent être éclairés par des tests menés sans a priori sur les « familles d'outils » rencontrés. Le pire étant de croire que seuls les outils positionnés comme « de curation » par leur éditeur permettent d'en faire. Trois principes pour parvenir à ce résultat : mener un veille sur les services émergents afin de rester ouvert aux nouvelles possibilités qu'ils offrent, les évaluer sur la base de critères fonctionnels et ergonomiques et les confronter à des logiques d'usage. • 


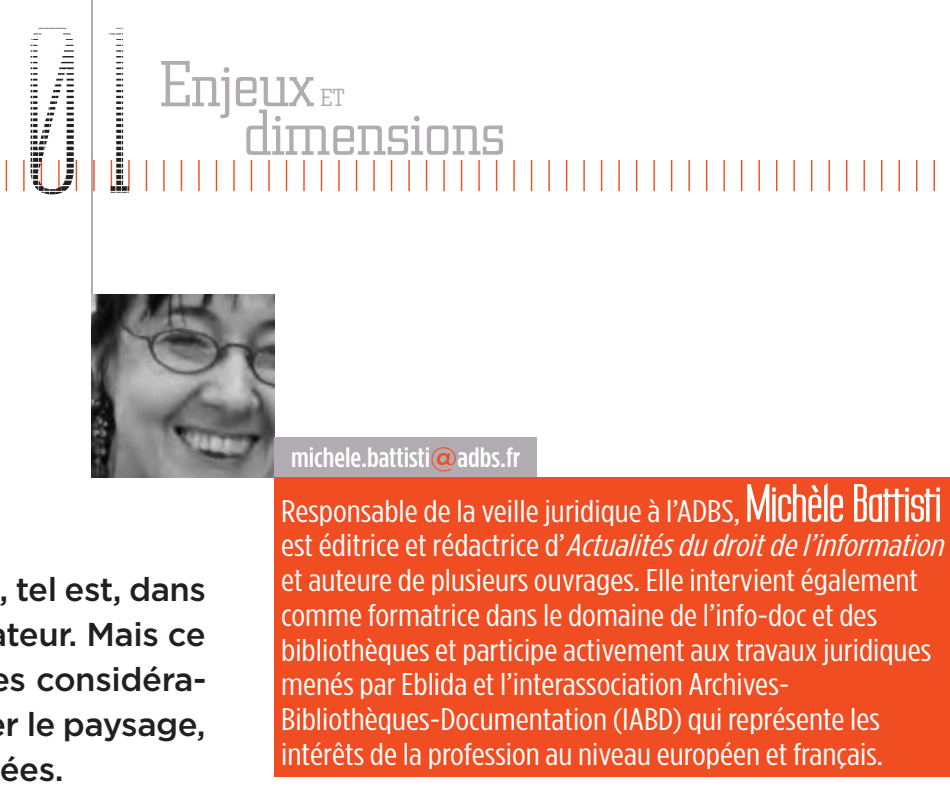

\section{La curation au risque du droit}

Après avoir sélectionné les œuvres, le curateur les diffuse à des communautés : telle est sa valeur ajoutée. Certes! Si ce n'est qu'aujourd'hui, c'est dans l'Internet qu'il puise sans se préoccuper des conditions légales de leur réutilisation. Or, seules les œuvres mises sous une licence autorisant le partage selon certaines conditions (de type Creative Commons) permettent en toute théorie ces pratiques.

Un article récent de cette revue $e^{1}$ rappelait que l'exception de courte citation ne s'applique pas aux photos sous forme de vignette, ni aux premières lignes d'un article non contextualisées dans une « œuvre seconde ", et que l'exception au droit d'auteur accordée aux revues de presse ne couvre que des pratiques journalistiques. Dans ce dernier cas, il s'agit naturellement de « panoramas de presse » redevables de droits.

Le lien hypertexte et les fonctions de partage sont indissociables des contenus ainsi agrégés. Le lien, certes, ne pose que rarement de problème, du moins lorsqu'il se borne à mener clairement vers la page d'origine ${ }^{2}$. Plus polémique serait, en revanche, le bouton de partage ou encore le repin (re-épingler) de Pinterest ${ }^{3}$ permettant une large dissémination.

\section{Diffuser sans autorisation expresse}

Sur Flickr, Picasa, YouTube, par exemple, on trouve des œuvres déposées par leurs auteurs ou avec leur accord. Du moins le présume-t-on. Une procédure de notification permet à tout auteur de signaler la présence illicite de leurs œuvres. L'hébergeur voit sa responsabilité engagée s'il ne bloque pas rapidement l'accès aux contenus contrefaisants ${ }^{4}$. Selon les conditions générales d'utilisation (CGU) des sites communautaires $^{5}$, donc les sites de curation, celui qui y « dépose » une œuvre cède, de manière non exclusive, le droit de la réutiliser. Cette disposition met en lumière un point particulièrement sensible : le curateur cède à des tiers des droits qu'il n'a pas.

Mais faire obstacle au partage des ouvres est possible techniquement. Dans ce cas, c'est l'auteur ou l'administrateur d'un site qui, en insérant les tags bloquant la réutilisation ${ }^{6}$, doit prendre des dispositions préventives. Quels droits pourra faire valoir l'auteur légitime qui n'aurait pas mis en œuvre de tels barrages ? Un tel renversement des responsabilités, s'il devait avoir lieu, serait intéressant à observer.

Les éditeurs eux-mêmes, tout en n'autorisant qu'un « usage strictement personnel, privé et non collectif ", favorisent la diffusion des premières lignes et des photos à des cercles, plus ou moins larges, via Facebook ou Google+, par exemple ${ }^{7}$. Les frontières entre les usages professionnels ou personnels, collectifs ou privés sont désormais totalement brouillées.

La rediffusion de l'œuvre porte-t-elle atteinte au modèle économique de l'auteur initial ? Le trafic généré par le lien estil suffisant $?^{8}$ : voilà certainement des questions essentielles qui vont se poser ! •

1 L. Maurel, « La curation met le droit dans tous ses états », Documentaliste-Sciences de l'information, $\mathrm{n}^{\circ} 2,2011$

2 M. Battisti, « Faut-il une autorisation pour faire un lien hypertexte ? », Paralipomènes, 11 décembre $2011 \mathrm{http}$ ://paralipomenes.net/wordpress/archives/6612 3 N. Jaimes, «Comment Pinterest est devenu le nouveau réseau social à la mode », Le Journal du Net, 22 février 2012

http://www.journaldunet.com/ebusiness/le-net/pinterest/

4 Cette responsabilité allégée sera remise en question si la société joue un rôle actif. C'est pour cette raison qu'Ebay vient d'être condamnée par la Cour d'appel de Paris, http://www.legalis.net/spip.php?page=breves-article\&id_article=3348

5 M. Lecardonnel, « Web 2.0: (GU et droit d’auteur », Expertises, n³ 363, nov. 2011 6 Benoit Marchal, « No-pin : que faire de Pinterest? ?, Le Déclencheur, 28 février 2012 http://www.declencheur.com/photo/carnet/note/no-pin-que-faire-de-pinterest 7 Le Monde, par exemple.

8 La légalité de Pinterest est sérieusement ébranlée. Alyson Shontell, "A Lawyer Who Is Also A Photographer Just Deleted All Her Pinterest Boards Out Of Fear", Business Insider, 28 Feb 2012, http://www.businessinsider.com/pinterest-copyright-issueslawyer-2012-2\#ixzz10QVe0AWJ 


\section{paint de vue}

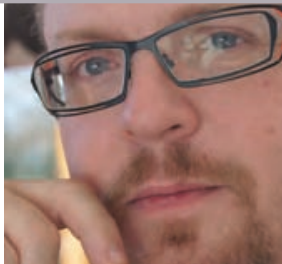

Frédéric Martinet est consultant indépendant. II a lancé Actulligence Consulting fin 2009 après huit années d'expériences professionnelles comme chargé de veille et responsable intelligence économique. Il accompagne aujourd'hui les entreprises dans la mise en place de leurs systèmes de veille concurrentielle, de veille sectorielle et de plus en plus fréquemment de veille image. II a créé et anime depuis 2001 Actulligence.com, un blog dédié aux professionnels de l'information et de l'e-réputation. de la curation ! Étoffer la matière première en la rapprochant d'autres informations, mettre en avant des sources d'informations primaires, des auteurs, aborder l'espace écran de façon éditoriale, valoriser certaines informations plus que d'autres... Imaginons même que le curateur apporte son propre commentaire à la dite information, ajoute son grain de savoir pour apporter une cohérence à sa « ligne éditoriale ». La curation vue et pratiquée comme cela, ce serait une aberration de dire qu'elle n'a pas d'intérêt !

Le problème, c'est que j'ai beau parcourir le Web, la curation ne ressemble pas à cela aujourd'hui. C'est plutôt, dans la grande majorité des cas, l'appropriation de contenus qui ne vous appartiennent pas à des fins de valorisation de sa propre marque. C'est du personal branding de bas étage où l'usurpateur occupe l'espace web en y déversant des contenus sous son propre label pour se mettre en avant. C'est principalement des solutions techniques qui, pour certaines d'entre elles, ont à cour de ne respecter aucun des fondements sains et intéressants de la curation telle que décrite.

Lautomatisation de la collecte d'information permet d'identifier les contenus sans fatigue. Les règles automatisées de publication rediffusent l'information sans que l'œil humain n'ait à la lire. Dans le meilleur des cas, le curateur n'aura qu'à cliquer pour publier le contenu dont le plus souvent il n'aura consulté qu'un court extrait et un titre.

Lespace est monolithique et uniforme, ne permettant que peu de variations dans l'agencement des contenus. Les auteurs sont rarement mis en avant et que dire des droits de rediffusion, bafoués dans la plupart des cas ? ${ }^{1}$. En rediffusant un article via une plateforme de curation, c'est le plus souvent le média ayant déjà relayé le contenu qui est cité comme auteur...

Tout est étudié pour générer une batterie de liens croisés, de contenus. Cette structure profite principalement à la plateforme de curation, dont les arguments trompent peu de monde : « Nous valorisons les auteurs », "Le contenu se doit d'être partagé, les droits d'auteurs c'est has been », « Nous générons du trafic sur le site des auteurs $» . .$.

Mensonges. Pour s'en convaincre, on lira les conditions générales d'utilisation de la plateforme de curation Scoop.it : toute idée que l'utilisateur publie sur le service ne lui appartient plus et Scoop.it ${ }^{2}$ se réserve le droit de l'utiliser comme il l'entend. ${ }^{3}$

Je ne peux donc cautionner cette forme de curation imposée par les vendeurs de plateforme qui n'ont pour seuls objectif et motivation de monétiser, avec des solutions premiums ou publicitaire, du contenu qui ne leur appartient pas. Je ne peux cautionner des sociétés dont le seul but est de profiter de ceux qui s'investissent pour rédiger, créer, réfléchir et partager. Je ne peux non plus cautionner les utilisateurs qui, sous couvert de manque de temps, participent à l'appauvrissement d'un idéal de partage pour promouvoir leur propre notoriété. •

1 Pour rappel, même les contenus sous Licence Creative Commons sont soumis à un certain formalisme de rediffusion, dont le rappel de la licence initiale.

2 Nulle volonté de stigmatiser un éditeur en particulier mais force est de constater que cette plateforme est représentative de tout ce que ne devrait pas être la curation. 3 Article 19: "You agree that any and all User Ideas are non-confidential and nonproprietary and will and need not be treated as such.", http://www.scoop.it/termsof-use 